\title{
Regularity of free boundary arising from optimal exercise of perpetual executive stock options
}

\author{
CONG QIN \\ Center for Financial Engineering, Soochow University, \\ 1 Shizi Street, Suzhou, Jiangsu 215006, P.R. China \\ E-mail:wacilee.qin@gmail.com \\ XINFU CHEN \\ Department of Mathematics, 301 Thackeray Hall, University of Pittsburgh, Pittsburgh, PA 15260, \\ USA \\ E-mail: xinfu@pitt.edu \\ XIN LAI \\ Department of Mathematics, Harbin Institute of Technology, \\ 92 Xidazhi Street, Harbin, Heilongjiang 150001, P.R. China \\ E-mail:laixin2002@163.com

\section{WANGHUI YU} \\ School of Mathematic Science, Soochow University, \\ 1 Shizi Street, Suzhou, Jiangsu 215006, P.R. China \\ E-mail: whyu@suda.edu.cn
}

[Received 15 February 2014 and in revised form 7 October 2014]

\begin{abstract}
In this paper we convert a variational inequality modeling perpetual executive stock options to a degenerate Stefan type free boundary problem where the free boundary is related to the boundary of optimal exercise and non-exercise regions. We establish the regularity of the free boundary by solving directly the resulting free boundary problem.

2010 Mathematics Subject Classification: Primary 35K86, 35K65, 91G80; Secondary 35Q93, 91G20.

Keywords: Free boundary problem, variational inequality, executive stock option, optimal exercise.
\end{abstract}

\section{Introduction}

Free boundaries are commonplace in nature. In mathematical finance it is important to find free boundaries since upon which optimal strategies are designed. In this paper, we study regularity of the free boundary of the variational inequality

$$
\min \{\mathfrak{Q}[\varphi], \mathbb{B} \varphi\}=0 \text { in } \mathbb{R} \times(0, \infty), \quad \varphi(\cdot, 0)=0,
$$

where $\varphi=\varphi(z, a)$

$$
\mathbb{Q}[\varphi]:=R a \varphi_{a}-\varphi_{z z}-v \varphi_{z}+\varphi_{z}^{2}, \quad \mathbb{B} \varphi:=\varphi_{a}-g^{+} .
$$


Here subscripts represent partial derivatives, $R>0$ and $v \in \mathbb{R}$ are constants, $g^{+}=\max \{g$, 0$\}$, and $g=g(z)=e^{z}-1$. Observe that the variable $a$ plays a role of time, so the variational inequality (1.1) is of parabolic type. We do not use the convention $t$ for the variable since the original problem itself admits a time variable.

As far as we know, problem (1.1) is a new type of free boundary problem that is not seen in the literature and deserves a systematic investigation, in the following sense:

1. The original full problem admits two time like variables, $t$ and $a$; here we study only the steady state problem, which happens to be a parabolic type free boundary problem (We leave the full double time problem as an open problem).

2. In the conventional free problems such as the solidification in physics and the American put option in mathematical finance, the first order time derivative of a solution of a variational inequality is the solution of a Stefan type free boundary problem. Here for the first time we obtain a variational inequality, for $\varphi$, whose "time" derivative, $\psi:=\varphi_{a}$, still satisfies a variational inequality; this is not a common phenomena that we have seen in classical free boundary problems. Here the second order "time" derivative, $w:=\varphi_{a a}$, satisfies a Stefan type free boundary problem.

3. We have a parabolic free boundary problem that degenerates at "time" $a=0$. Only through a deep rigorous mathematical analysis (instead of financial intuition) can we discover how initial conditions should be imposed, for $\varphi, \psi$, and $w$, respectively.

4. Note that by introducing a variable $s=\ln a$, we have a variational inequality problem

$$
\min \left\{R \varphi_{s}-\varphi_{z z}-v \varphi_{z}+\varphi_{z}^{2}, \varphi_{s}-e^{s} g^{+}\right\}=0 \quad \text { for } z \in \mathbb{R}, s \in \mathbb{R} .
$$

This is by no means a conventional parabolic free boundary problem. From a dynamical system point of view, we are dealing with finding an unstable manifold in infinite space dimension, coupled with inhomogeneity and free boundaries. Here even finding the initial (at $s=-\infty)$ position of the free boundary is a challenge.

We shall develop a systematic method to deal with such a problem, based on new and conventional techniques.

Under the condition $R>\max \{0, v+1\}$, Song and $\mathrm{Yu}[16]$ established the existence of a strong solution and in our companion paper [10], we established the existence of a unique classical solution and the existence of a continuous and strictly decreasing function $s(\cdot)$ defined on $[0, \infty)$ such that

$$
\begin{array}{lll}
\mathbb{Q}[\varphi]=0<B \varphi & \text { in } \quad \mathbf{N}:=\{(z, a) \mid a>0, z<s(a)\}, \\
\mathbb{Q}[\varphi]>0=B \varphi & \text { in } \quad \mathbf{T}:=\{(z, a) \mid a>0, z>s(a)\}, \\
\mathbb{Q}[\varphi]=0=B \varphi & \text { on } \quad \Gamma:=\{(z, a) \mid a>0, z=s(a)\} .
\end{array}
$$

We call the graph $z=s(a)$ the free boundary. In its original context, $\mathbf{N}$ is called no-trading region and $\mathbf{T}$ the trading region, and optimal strategies depend only on the free boundary. Here in this paper we shall use a free boundary approach proving that $s \in C^{\frac{3}{2}}([0, \infty)) \cap C^{\infty}((0, \infty))$.

Problem (1.1) originates from Rogers and Scheinkman [15] where they study Executive Stock Options (ESOs) which are American call options granted by a firm with long maturity to an executive as a form of benefit in addition to salary. An American call option is a right but not an obligation to buy a share of stock at a fixed price at any time before expiry. The option is called perpetual if there is no expiry. For option holder, the problem is to find optimal exercise strategy. An exercise strategy can be described mathematically by an adaptive stochastic process $\{A(t)\}_{t \geqslant 0}$ 
where $A(t)$ is right-continuous, decreasing, non-negative, and $A\left(0^{-}\right)=\mathrm{A}$; here $A(t)$ is the number of ESO's holding at time $t$ (after trading), and A is the initial number of shares of ESOs. There are a number of studies for ESOs; see Lambert, Larcker and Verechia [12], Carpenter [1], Hall and Murphy [7], Ingersoll [8], Jain and Subramanian [9], Grasselli and Henderson [6], Leung and Sircar [13], Song and Yu [16, 17]. The paper [2] by Carpenter, Stanton, and Wallace contains an extensive list of references. Based on the models proposed in $[15,16]$ and our analysis presented in $[10,14]$, in this paper we continue the study of the value function

$$
V(x, s, \mathrm{~A}):=\lim _{T \rightarrow \infty} \sup _{\{A(t)\} \in \mathbb{Q}} \mathbb{E}\left[U\left(x-\int_{0}^{T} e^{-r t}[S(t)-K]^{+} d A(t)\right) \mid S(0)=s\right] .
$$

Here, $x, s, K$, A are initial cash, stock price, strike price, and ESO holding respectively, $r \geqslant 0$ is a constant discount rate, $U$ is a given utility (concave increasing) function, and $Q$ is the set of all admissible strategies. Assume that the stock price $S(t)$ follows Black-Scholes model:

$$
S(t)=s e^{\left(\alpha-\frac{\sigma^{2}}{2}\right) t+\sigma W(t)} \quad \text { for } \quad t \geqslant 0
$$

where $\{W(t)\}_{t \geqslant 0}$ is the standard Brownian motion, $\alpha$ is constant expected return rate, and $\sigma>0$ is constant volatility ${ }^{1}$.

One can derive (c.f. [14]) that $V$ is a viscosity solution of

$$
\min \left\{r A V_{A}-\frac{1}{2} \sigma^{2} s^{2} V_{s s}-\alpha s V_{s}, \quad V_{A}-(s-K)^{+} V_{x}\right\}=0 \text { in }(0, \infty)^{3},\left.\quad V\right|_{A=0}=U(x) .
$$

Using exponential utility function, i.e., $U(z)=-e^{-\gamma z}$, where $\gamma$ is a positive constant, and

$$
z=\log \frac{s}{K}, \quad a=\gamma K A, \quad R=\frac{2 r}{\sigma^{2}}, \quad v=\frac{2}{\sigma^{2}}\left[\alpha-\frac{\sigma^{2}}{2}\right],
$$

one can show that $V(x, s, A)=e^{-\gamma x} u(z, a)$ where $u$ solves

$$
\min \left\{\operatorname{Rau}_{a}-u_{z z}-v u_{z}, u_{a}+g^{+} u\right\}=0 \text { in } \mathbb{R} \times(0, \infty), \quad u(\cdot, 0)=-1 .
$$

The dimensionless certainty equivalent is a function $\varphi=\varphi(z, a)$ such that

$$
V=U\left(x+\gamma^{-1} \varphi\right)
$$

Then $\varphi=-\ln (-u)$ and is a viscosity solution of (1.1). Using a technique in [3], one can show that $V$ is concave in $(x, A)$ and $\varphi$ is concave in $a$.

In [14], we have shown that $\varphi=\infty$ if and only if $r \leqslant \alpha-\frac{\sigma^{2}}{2}$ (i.e., the discount rate is no bigger than the growth rate of the stock). In addition, we show that when $r>\alpha-\frac{\sigma^{2}}{2}$, the optimal exercise strategy is to hold the following amount of option at time $t$ :

$$
A^{\text {optimal }}(t)=\min \left\{\mathrm{A}, \min _{0 \leqslant \rho \leqslant t} \frac{e^{r \rho} a_{*}\left(\ln \frac{S(\rho)}{K}\right)}{\gamma K}\right\},
$$

\footnotetext{
1 Although the geometrical Brownian motion model for the stock price faces many critics in recent years, it is by far the mostly used in analysis and in practice and many important mathematical finance theories depend on this kind of models. In our opinion, this model is by no means worse than any other models such as the jump-diffusion process model (since it contains many parameters that are very hard to measure by one sample historical curve).
} 
where $a=a_{*}(z)$ is the inverse function of $z=s(a)$, with natural extension $a_{*}=\infty$ for $z \leqslant 0=$ $s(\infty)$ and $a_{*}=0$ for $z>s(0)$. Also, explicit solutions are given for the case $r=0$ and the case $K=0$ respectively. These explicit solutions indicate that in general $s(0)<\infty$ if and only if $\alpha<r$, which is equivalent to $R>1+v$. In this paper, we always assume that $R>\max \{0,1+v\}$. In a subsequent paper [11], we shall consider the remaining case $v<R \leqslant v+1$.

In [16], Song and Yu study (1.2), established the existence of a strong solution.

In [10] we discover that the function $\psi:=\varphi_{a}$ satisfies the following variational inequality:

$$
\min \{\mathfrak{F}[\psi], \psi-g\}=0 \quad \text { in } \mathbb{R} \times[0, \infty),
$$

where $\mathcal{F}[\psi]:=R a \psi_{a}+R \psi-\psi_{z z}+\left(2 \int_{0}^{a} \psi_{z}(z, t) d t-v\right) \psi_{z}$. We remark that the function $g^{+}$ in the formulation (1.1) can be replaced by $g$ since one never exercises when stock price is below strike price; see [4]. Also, (1.3) at $a=0$ provides the equation for the initial value $\psi_{0}:=\psi(\cdot, 0)=$ $\varphi_{a}(\cdot, 0)$ :

$$
\min \left\{R \psi_{0}-\psi_{0}^{\prime \prime}-v \psi_{0}^{\prime}, \quad \psi_{0}-g\right\}=0 \text { in } \mathbb{R} .
$$

There is a unique solution given by

$$
\psi_{0}(z):=\left\{\begin{array} { l l } 
{ g ( z ) } & { \text { if } z \geqslant b , } \\
{ g ( b ) e ^ { \lambda ( z - b ) } } & { \text { if } z < b , }
\end{array} \quad \left\{\begin{array}{l}
\lambda:=\frac{\sqrt{v^{2}+4 R}-v}{2}, \\
b:=\ln \frac{\lambda}{\lambda-1} .
\end{array}\right.\right.
$$

Here the condition $R>\max \{0, v+1\}$ guarantees that $\lambda>1$, so $b$ is well-defined. By establishing a strong solution of (1.3), we obtain a classical solution of (1.1). Also, we show that $s(\infty)=0$.

In this paper we work on the function $w:=\psi_{a}=\varphi_{a a}$. In $\mathbf{N}$ differentiating $\mathcal{F}[\psi]=0$ with respect to $a$ we obtain $R a w_{a}+2 R w-w_{z z}-\left[v-2 \varphi_{z}\right] w_{z}+2 \psi_{z}^{2}=0$. Setting $a=0$ we find a system for $w_{0}:=w(\cdot, 0)$, deriving that

$$
\left.w_{0}(z):=g^{2}(b)\left\{e^{2 \lambda[z-b]}-e^{\Lambda(2 R)[z-b]}\right\} \mathbf{1}_{\{z \leqslant b}\right\}
$$

where $\Lambda(x)$ is the positive root of $\Lambda^{2}+v \Lambda=x$. By differentiating the relations $\psi(s(a), a)=$ $g(s(a))$ and $\psi_{z}(s(a), a)=g^{\prime}(s(a))$ with respect to $a$, we obtain the following Stefan type free boundary problem:

$$
\begin{cases}\left(R a w+2 \varphi_{z} \psi_{z}\right)_{a}=w_{z z}+v w_{z}-R w & \forall a \geqslant 0, z<s(a), \\ w=0 & \forall a \geqslant 0, z \geqslant s(a), \\ w_{z}=\left[g^{\prime \prime}+\left(v-2 \varphi_{z}\right) g^{\prime}-R g\right] s^{\prime} & \forall a \geqslant 0, z=s(a)^{-}, \\ \psi_{a}=w, \quad \varphi_{a}=\psi & \forall a \geqslant 0, z \in \mathbb{R}, \\ w(\cdot, 0)=w_{0}, \psi(\cdot, 0)=\psi_{0}, \varphi(\cdot, 0) \equiv 0, s(0)=b .\end{cases}
$$

If $\varphi$ and $\psi$ are known functions, (1.6) can be viewed as a one-phase Stefan problem (c.f. [5]) modeling solidification of undercooled liquid, where $w$ is the temperature function, the region $(-\infty, s(a))$ in which $w<0$ is the region of undercooled liquid at time $a$, the region $(s(a), \infty)$ in which $w=0$ is the solid region, and $l(z, a):=\operatorname{Rg}(z)+\left[2 \varphi_{z}(z, a)-v\right] g^{\prime}(z)-g^{\prime \prime}(z)$ is the latent heat, the energy needed to melt unit amount of solid at the melting temperature, at location $z$, 
and at time $a$. Different from the classical and well-studied Stefan problem, here we have a coupled system, variable latent heat not a priori known to be positive, and degeneracy at $a=0$. In addition, it is known that solidification of undercooled liquid is often an unstable process. Nevertheless, due to its original variational nature, we shall develop new techniques to solve the problem, showing that the free boundary problem is well-posed. Our main result is the following:

THEOREM 1 Let $R$ and $v$ be constants satisfying $R>\max \{0,1+v\}, g(z)=e^{z}-1$, and $\left(b, \psi_{0}, w_{0}\right)$ be defined in (1.4) and (1.5). Then (1.6) admits a classical solution $(\varphi, \psi, w, s)$ satisfying

$$
s \in C^{3 / 2}([0, \infty)) \cap C^{\infty}((0, \infty)), \quad \varphi, \psi, w \in C^{2,1}(\bar{D}) \cap C^{\infty}(D),
$$

where $D:=\{(z, a) \mid a>0, z \leqslant s(a)\}$. In addition, $\varphi \in C^{2}(\mathbb{R} \times[0, \infty))$ is the unique solution of (1.1), $\psi>g$ in $\mathbf{N}:=\{(z, a) \mid a>0, z<s(a)\}, \psi \equiv g$ in $\mathbf{N}^{c}, s^{\prime}<0<s \leqslant b$ on [0,, , and across $\boldsymbol{\Gamma}:=\{(s(a), a) \mid a>0\}$,

$$
\begin{gathered}
\llbracket \varphi \rrbracket=0, \quad \llbracket \varphi_{z} \rrbracket=0, \quad \llbracket \varphi_{a} \rrbracket=0, \quad \llbracket \varphi_{z z} \rrbracket=0, \quad \llbracket \varphi_{a z} \rrbracket=0, \quad \llbracket \varphi_{a a} \rrbracket=0, \\
\llbracket \varphi_{a z z} \rrbracket=-\ell, \quad \llbracket \varphi_{a a z} \rrbracket=\ell s^{\prime}, \quad \llbracket \varphi_{a a a} \rrbracket=-\ell s^{\prime 2},
\end{gathered}
$$

where $\llbracket f \rrbracket:=f\left(s(a)^{+}, a\right)-f\left(s(a)^{-}, a\right)$ and $\ell:=e^{s(a)}\left[2 \varphi_{z}(s(a), a)+R-1-v\right]-R>0$ for $a \geqslant 0$. Moreover,

$$
\llbracket \varphi_{z z z} \rrbracket=\frac{\ell(a)}{s^{\prime}(a)}, \quad \varphi_{z z z}\left(b^{+}, a\right)-\varphi_{z z z}\left(b^{-}, a\right)=-\frac{\ell(0)}{s^{\prime}(0)}=\frac{\lambda^{2}(\lambda-1)^{2}}{2 \lambda-\Lambda(2 R)} \quad \forall a>0 .
$$

Here the improved regularity $\varphi \in C^{2}(\mathbb{R} \times(0, \infty))$ from $\varphi \in C^{2,1}$ in [10] for the underline parabolic free boundary problem is due to the fact that we obtain the new estimate for $\psi=\varphi_{a} \in$ $C^{2,1}(\bar{D})$.

Note that the jumps of $\varphi_{z z z}$ on $\boldsymbol{\Gamma}$ and on $\{b\} \times[0, \infty)$ cancels at $a=0$, compatible with $\varphi(\cdot, 0) \equiv 0$.

The rest of the paper is organized as follows. In Section 2 we first derive the differential equations, initial conditions, and free boundary conditions for $\psi$ and $w$. Since the linear second order parabolic operator $R a \partial_{a}-\partial_{z z}$ is degenerate at $a=0$, we shall first study (1.6) in time interval $a \in[\varepsilon, \infty)(0<\varepsilon \ll 1)$. For this we construct carefully compatible initial values at $a=\varepsilon$ for the approximation problem. In Section 3 we establish local (in time $a \in[\varepsilon, T], 0<T-\varepsilon \ll 1$ ) existence of the initial value problem with initial values given at $a=\varepsilon$. In Section 4 we establish a priori estimate for the approximate solution. Finally in Section 5 we first establish global (in time $a \in[\varepsilon, \infty)$ ) existence of the solution of the approximate problem and then send $\varepsilon \searrow 0$ to obtain the solution of (1.6) and prove Theorem 1.

In the sequel, we use the convention that all functions are continuous from the left-hand side, i.e., $f(z)=\lim _{x \nearrow z} f(x)$. Also, $O(f)=O(1) f$ where $O(1)$ is a generic quantity bounded by a constant $M$ and $M$ stands for a generic positive constant depending only on $R$ and $v$.

\section{Basic equations}

In this section we derive free boundary and initial conditions for the functions

$$
\psi:=\varphi_{a}, \quad w:=\psi_{a}=\varphi_{a a}, \quad v:=w_{a}=\psi_{a a}=\varphi_{a a} .
$$

Also, we construct approximation for these functions and the free boundary at time $a=\varepsilon$. 


\subsection{The differential equations}

The equation for $\varphi$ and their derivatives with resect to $a$ in $\mathbf{N}$ can be expressed as

$$
\begin{aligned}
\operatorname{Ra} \varphi_{a} & =\varphi_{z z}+\left(v-\varphi_{z}\right) \varphi_{z} \\
\operatorname{Ra} \psi_{a} & =\psi_{z z}+\left(v-2 \varphi_{z}\right) \psi_{z}-R \psi \\
\operatorname{Raw}_{a} & =w_{z z}+\left(v-2 \varphi_{z}\right) w_{z}-2 R w-2 \psi_{z}^{2}, \\
\operatorname{Rav}_{a} & =v_{z z}+\left(v-2 \varphi_{z}\right) v_{z}-3 R v-6 \psi_{z} w_{z}
\end{aligned}
$$

\subsection{The free boundary conditions}

On the free boundary $\boldsymbol{\Gamma}:=\{(s(a), a) \mid a>0\}$, we can differentiate the basic relations $\psi-g=0$ and $\psi_{z}-g^{\prime}=0$ to obtain $w=0$ and $w_{z}+\left(\psi_{z z}-g^{\prime \prime}\right) s^{\prime}=0$. Differentiating the resulting equations again we obtain $v+w_{z} s^{\prime}=0$ and $v_{z}+\left[w_{z z}+\left(\psi_{z z}-g^{\prime \prime}\right)^{\prime}\right] s^{\prime}+\left(\psi_{z z}-g^{\prime \prime}\right) s^{\prime \prime}=0$. Hence, on the free boundary $z=s(a)$, we have the following three sets of free boundary conditions:

$$
\begin{aligned}
& \left\{\begin{array}{l}
\psi(s(a), a)=g(s(a)), \\
\psi_{z}(s(a), a)=g^{\prime}(s(a)), \\
\ell(a):=\psi_{z z}(s(a), a)-g^{\prime \prime}(s(a)) ;
\end{array}\right. \\
& \left\{\begin{array}{l}
w(s(a), a)=0, \\
w_{z}(s(a), a)=-\ell(a) s^{\prime}(a) ;
\end{array}\right. \\
& \left\{\begin{array}{l}
v(s(a), a)=\ell(a) s^{\prime}(a)^{2}, \\
v_{z}(s(a), a)=-\ell(a) s^{\prime \prime}(a)-\left\{w_{z z}(s(a), a)+\ell^{\prime}(a)\right\} s^{\prime}(a) .
\end{array}\right.
\end{aligned}
$$

The system for $w$ is a Stefan type free boundary problem modeling one phase solidification of undercooled liquid, where $\ell$ is the latent heat. Using the differential equation and boundary conditions for $\psi$, the latent heat can be written as

$$
\begin{aligned}
\ell(a) & =\psi_{z z}(s(a), a)-g^{\prime \prime}(s(a)) \\
& =R a w+\left(2 \varphi_{z}-v\right) \psi_{z}+R \psi-\left.g^{\prime \prime}\right|_{z=s(a)}=l(s(a), a), \\
l(z, a) & :=e^{z}\left[2 \varphi_{z}(z, a)+R-1-v\right]-R .
\end{aligned}
$$

\subsection{The initial conditions for $(\psi, w, v)$}

Let $b:=s(0), \psi_{0}:=\psi(\cdot, 0), w_{0}:=w(\cdot, 0)$ and $v_{0}:=v(\cdot, 0)$. Using the initial condition $\varphi(\cdot, 0) \equiv$ 0 , the differential equations for $(\psi, w, v)$ at $a=0$, and the free boundary conditions at $z=s(0)$, we obtain

$$
\begin{array}{lll}
\psi_{0}^{\prime \prime}+v \psi_{0}^{\prime}-R \psi_{0}=0 & \text { in }(-\infty, b), & \psi_{0}(b)=g(b), \psi_{0}^{\prime}(b)=g^{\prime}(b), \\
w_{0}^{\prime \prime}+v w_{0}^{\prime}-2 R w_{0}=2 \psi_{0}^{\prime 2} & \text { in }(-\infty, b), & w_{0}(b)=0, \\
v_{0}^{\prime \prime}+v v_{0}^{\prime}-3 R v_{0}=6 \psi_{0}^{\prime} w_{0}^{\prime} & \text { in }(-\infty, b), & v_{0}(b)=\frac{w_{0}^{\prime}(b)^{2}}{\psi_{0}^{\prime \prime}(b)-g^{\prime \prime}(b)} .
\end{array}
$$


These linear problems are supplemented by the boundary conditions $\psi_{0}(-\infty)=0, w_{0}(-\infty)=0$, and $v_{0}(-\infty)=0$. For convenience, we denote by $\Lambda(x)$ the unique positive root of $\Lambda^{2}+v \Lambda=$ $x>0$, i.e.,

$$
\Lambda(x)=\frac{\sqrt{v^{2}+4 x}-v}{2} .
$$

It is easy to see that $\Lambda^{\prime}>0>\Lambda^{\prime \prime}$ so for every $x>0, y>0, z>0, x \neq z$, and $\theta \in(0,1)$,

$$
\Lambda(x+y)<\Lambda(x)+\Lambda(y), \quad \Lambda(\theta x+[1-\theta] z)>\theta \Lambda(x)+[1-\theta] \Lambda(z) .
$$

For simplicity, we set $\lambda=\Lambda(R), \lambda_{2}=\Lambda(2 R), \lambda_{3}=\Lambda(3 R)$. Under the condition $R>\max \{0,1+$ $v\}$, one can check that $\lambda>1$. Then we can derive that

$$
\begin{cases}\psi_{0}(z)=g(b) e^{\lambda(z-b)} \quad\left(\lambda g(b)=g^{\prime}(b) \Rightarrow e^{b}=\frac{\lambda}{\lambda-1}\right) & \forall z \leqslant b, \\ w_{0}(z)=g^{2}(b)\left\{e^{2 \lambda(z-b)}-e^{\lambda_{2}(z-b)}\right\} & \forall z \leqslant b, \\ v_{0}(z)=g^{3}(b)\left\{2 e^{3 \lambda(z-b)}-3 e^{\left(\lambda+\lambda_{2}\right)(z-b)}+\left[\frac{\left(2 \lambda-\lambda_{2}\right)^{2}}{\lambda(\lambda-1)}+1\right] e^{\lambda_{3}(z-b)}\right\} & \forall z \leqslant b .\end{cases}
$$

When $z>b, \psi_{0} \equiv g, w_{0} \equiv 0$, and $v_{0} \equiv 0$. Note that $v_{0}$ is not continuous at $b$. Also,

$$
\begin{gathered}
\ell(0)=\psi_{0}^{\prime \prime}(b)-g^{\prime \prime}(b)=\lambda, \\
s(0)=b=\ln \frac{\lambda}{\lambda-1}, \\
s^{\prime}(0)=-\frac{w_{0}^{\prime}(b)}{\ell(0)}=-\frac{v_{0}(b)}{w_{0}^{\prime}(b)}=\frac{\Lambda(2 R)-2 \lambda}{(\lambda-1)^{2} \lambda}<0, \\
\psi_{0}>g^{+}, \quad 0<\psi_{0}^{\prime}=g^{\prime} e^{(\lambda-1)(z-b)}, \quad w_{0}<0 \quad \text { in }(-\infty, b) .
\end{gathered}
$$

\subsection{Approximation at $a=\varepsilon$}

Since the differential equation for $w$ is degenerate at $a=0$, we shall first solve the problem in time interval $a \in[\varepsilon, \infty)$ and then let $\varepsilon \searrow 0$. Here we define approximations of $(\varphi, \psi, w, v, s)$ at $a=\varepsilon$, denoted by $\left(\varphi_{0}^{\varepsilon}, \psi_{0}^{\varepsilon}, w_{0}^{\varepsilon}, v_{0}^{\varepsilon}, s_{\varepsilon}\right)$.

Lemma 2.1 Let $\left(\psi_{0}, w_{0}, v_{0}\right)$ be defined in (2.1). For each $\varepsilon>0$ define, on $(-\infty, b]$,

$$
\begin{aligned}
\varphi_{0}^{\varepsilon} & :=\varepsilon \psi_{0}+\frac{1}{2} \varepsilon^{2} w_{0}+\frac{1}{6} \varepsilon^{3} v_{0}, \\
\psi_{0}^{\varepsilon} & :=\frac{1}{\varepsilon R}\left(\varphi_{0}^{\varepsilon \prime \prime}+v \varphi_{0}^{\varepsilon \prime}-\varphi_{0}^{\varepsilon \prime 2}\right), \\
w_{0}^{\varepsilon} & :=\frac{1}{\varepsilon R}\left(\psi_{0}^{\varepsilon^{\prime \prime}}+\left[\nu-2 \varphi_{0}^{\varepsilon \prime}\right] \psi_{0}^{\varepsilon^{\prime}}-R \psi_{0}^{\varepsilon}\right), \\
v_{0}^{\varepsilon} & :=\frac{1}{\varepsilon R}\left(w_{0}^{\varepsilon \prime \prime}+\left[\nu-2 \varphi_{0}^{\varepsilon \prime}\right] w_{0}^{\varepsilon^{\prime}}-2 R w_{0}^{\varepsilon}-2 \psi_{0}^{\varepsilon / 2}\right) .
\end{aligned}
$$

Then

$$
\psi_{0}^{\varepsilon}=\psi_{0}+\varepsilon w_{0}+\frac{1}{2} \varepsilon^{2} v_{0}+\varepsilon^{3} u_{3}^{\varepsilon}, \quad w_{0}^{\varepsilon}=w_{0}+\varepsilon v_{0}+\varepsilon^{2} u_{2}^{\varepsilon}, \quad v_{0}^{\varepsilon}=v_{0}+\varepsilon u_{1}^{\varepsilon},
$$


where $u_{i}^{\varepsilon}, i=1,2,3$ are smooth functions on $(-\infty, b]$ satisfying

$$
\left|\frac{d^{j}}{d z^{j}} u_{i}^{\varepsilon}(z)\right| \leqslant M\left[1+\varepsilon^{8}\right] e^{[\lambda+\Lambda(3 R)][z-b]} \quad \forall z \leqslant b, i, j=1,2,3,
$$

where $M$ is a positive constant depending only on $v$ and $R$.

Proof. The assertion follows by direct evaluation and the fact that $2 \Lambda(2 R)>\lambda+\Lambda(3 R)$.

\subsection{Compatibility conditions at the corner $\left(s_{\varepsilon}(\varepsilon), \varepsilon\right)$ - modification of $g$}

Denote by $\left(s_{0}^{\varepsilon}, s_{1}^{\varepsilon}\right)$ the approximation of $\left(s(\varepsilon), s^{\prime}(\varepsilon)\right)$, we shall modify $g$ to $g_{\varepsilon}$ to enforce the following compatibility conditions:

$$
w_{0}^{\varepsilon}\left(s_{0}^{\varepsilon}\right)=0, \quad \psi_{0}^{\varepsilon}\left(s_{0}^{\varepsilon}\right)=g_{\varepsilon}\left(s_{0}^{\varepsilon}\right), \quad \psi_{0}^{\varepsilon^{\prime}}\left(s_{0}^{\varepsilon}\right)=g_{\varepsilon}^{\prime}\left(s_{0}^{\varepsilon}\right), \quad s_{1}^{\varepsilon}=-\frac{v_{0}^{\varepsilon}\left(s_{0}^{\varepsilon}\right)}{w_{0}^{\varepsilon^{\prime}}\left(s_{0}^{\varepsilon}\right)}=\frac{w_{0}^{\varepsilon^{\prime}}\left(s_{0}^{\varepsilon}\right)}{g_{\varepsilon}^{\prime \prime}\left(s_{0}^{\varepsilon}\right)-\psi_{0}^{\varepsilon^{\prime \prime}}\left(s_{0}^{\varepsilon}\right)}
$$

The equation $w_{0}^{\varepsilon}\left(s_{0}^{\varepsilon}\right)=0$ determines a unique $s_{0}^{\varepsilon}$. Consequently, $s_{1}^{\varepsilon}$ is uniquely determined. For other compatibility conditions, we need

$$
g_{\varepsilon}\left(s_{0}^{\varepsilon}\right)=\psi_{0}^{\varepsilon}\left(s_{0}^{\varepsilon}\right), \quad g_{\varepsilon}^{\prime}\left(s_{0}^{\varepsilon}\right)=\psi_{0}^{\varepsilon^{\prime}}\left(s_{0}^{\varepsilon}\right), \quad g_{\varepsilon}^{\prime \prime}\left(s_{0}^{\varepsilon}\right)=\psi_{0}^{\varepsilon^{\prime \prime}}\left(s_{0}^{\varepsilon}\right)-\frac{w_{0}^{\varepsilon^{\prime}}\left(s_{0}^{\varepsilon}\right)^{2}}{v_{0}^{\varepsilon}\left(s_{0}^{\varepsilon}\right)} .
$$

Working in the category of $g_{\varepsilon}(z)=c_{1}+c_{2} e^{1_{\varepsilon} z}$, we define

$$
1_{\varepsilon}:=\frac{\psi_{0}^{\varepsilon^{\prime \prime}}\left(s_{0}^{\varepsilon}\right)}{\psi_{0}^{\varepsilon^{\prime}}\left(s_{0}^{\varepsilon}\right)}-\frac{w_{0}^{\varepsilon^{\prime}}\left(s_{0}^{\varepsilon}\right)^{2}}{v_{0}^{\varepsilon}\left(s_{0}^{\varepsilon}\right) \psi_{0}^{\varepsilon^{\prime}}\left(s_{0}^{\varepsilon}\right)}, \quad g_{\varepsilon}(z):=\psi_{0}^{\varepsilon}\left(s_{0}^{\varepsilon}\right)+\frac{\psi_{0}^{\varepsilon^{\prime}}\left(s_{0}^{\varepsilon}\right)}{1_{\varepsilon}}\left(e^{1_{\varepsilon}\left[z-s_{0}^{\varepsilon}\right]}-1\right)
$$

LEMMA 2.2 For each sufficiently small positive $\varepsilon$, there exists a unique $s_{0}^{\varepsilon}<b$ such that $w_{0}^{\varepsilon}\left(s_{0}^{\varepsilon}\right)=$ $0, w_{0}^{\varepsilon}<0$ in $\left(-\infty, s_{0}^{\varepsilon}\right)$ and $w_{0}^{\varepsilon}>0$ in $\left(s_{0}^{\varepsilon}, b\right]$.

Proof. When $\varepsilon$ is small, $e^{-\Lambda(2 R)[z-b]} g^{-2}(b) w_{0}^{\varepsilon}(z)=e^{[2 \lambda-\Lambda(2 R)][z-b]}-1+O(\varepsilon)$ is negative when $z<0$, strictly increasing on $[0, b]$, and positive at $b$ (since $w_{0}(b)=0<v_{0}(b)$ ); hence, there exists a unique $s_{0}^{\varepsilon} \in(0, b)$ such that $w_{0}^{\varepsilon}\left(s_{0}^{\varepsilon}\right)=0<w_{0}^{\varepsilon^{\prime}}\left(s_{0}^{\varepsilon}\right), w_{0}^{\varepsilon}<0$ in $\left(-\infty, s_{0}^{\varepsilon}\right)$ and $w_{0}^{\varepsilon}>0$ in $\left(s_{0}^{\varepsilon}, b\right]$.

LEMMA 2.3 Let $g_{\varepsilon}$ be defined as in (2.3) and $s_{1}^{\varepsilon}:=-v_{0}^{\varepsilon}\left(s_{0}^{\varepsilon}\right) / w_{0}^{\varepsilon^{\prime}}\left(s_{0}^{\varepsilon}\right)$. Then (2.2) holds. In addition, as $\varepsilon \searrow 0$, we have the following asymptotics:

$$
\begin{gathered}
s_{0}^{\varepsilon}=s(0)+s^{\prime}(0) \varepsilon+O\left(\varepsilon^{2}\right), \quad s_{1}^{\varepsilon}=s^{\prime}(0)+O(\varepsilon), \quad 1_{\varepsilon}=1+O(\varepsilon), \\
g_{\varepsilon}\left(s_{0}^{\varepsilon}\right)=g\left(s_{0}^{\varepsilon}\right)+O\left(\varepsilon^{3}\right), \quad g_{\varepsilon}^{\prime}\left(s_{0}^{\varepsilon}\right)=g^{\prime}\left(s_{0}^{\varepsilon}\right)+O\left(\varepsilon^{2}\right), \quad g_{\varepsilon}^{\prime \prime}\left(s_{0}^{\varepsilon}\right)=\psi_{0}^{\varepsilon}\left(s_{0}^{\varepsilon}\right)^{\prime \prime}-\lambda+O(\varepsilon) .
\end{gathered}
$$

Proof. It is clear by the definition of $s_{1}^{\varepsilon}$ and $g_{\varepsilon}$ that (2.2) holds. Since $w_{0}^{\varepsilon}=w_{0}+\varepsilon v_{0}+O\left(\varepsilon^{2}\right)$, by 
Taylor expansion, $s_{0}^{\varepsilon}=b-\varepsilon \frac{v_{0}(b)}{w_{0}^{\prime}(b)}+O\left(\varepsilon^{2}\right)=s(0)+s^{\prime}(0) \varepsilon+O\left(\varepsilon^{2}\right)$. Also,

$$
\begin{aligned}
g_{\varepsilon}\left(s_{0}^{\varepsilon}\right) & =\psi_{0}^{\varepsilon}\left(s_{0}^{\varepsilon}\right) \\
& =\psi_{0}\left(s_{0}^{\varepsilon}\right)+\varepsilon w_{0}\left(s_{0}^{\varepsilon}\right)+\frac{\varepsilon^{2}}{2} v_{0}\left(s_{0}^{\varepsilon}\right)+O\left(\varepsilon^{3}\right) \\
& =g\left(s_{0}^{\varepsilon}\right)+\left[\psi_{0}\left(s_{0}^{\varepsilon}\right)-g\left(s_{0}^{\varepsilon}\right)\right]+\varepsilon\left[w_{0}(b)+w_{0}^{\prime}(b)\left(s_{0}^{\varepsilon}-b\right)\right]+\frac{1}{2} \varepsilon^{2} v_{0}(b)+O\left(\varepsilon^{3}\right) \\
& =g\left(s_{0}^{\varepsilon}\right)+\frac{\varepsilon^{2}}{2}\left(\left[\psi_{0}^{\prime \prime}(b)-g^{\prime \prime}(b)\right] s^{\prime}(0)^{2}+2 w_{0}^{\prime}(b) s^{\prime}(0)+v_{0}(b)\right)+O\left(\varepsilon^{3}\right) \\
& =g\left(s_{0}^{\varepsilon}\right)+O\left(\varepsilon^{3}\right)
\end{aligned}
$$

since $v_{0}(b)=-w_{0}^{\prime}(b) s^{\prime}(0)$ and $w_{0}^{\prime}(b)=-\left(\psi_{0}^{\prime \prime}(b)-g^{\prime \prime}(b)\right) s^{\prime}(0)$. The other asymptotics can be similarly proven.

Lemma 2.4 When $0<\varepsilon \ll 1, \psi_{0}^{\varepsilon^{\prime}}(z)<g_{\varepsilon}^{\prime}(z) e^{\left[\lambda-1_{\varepsilon}\right]\left[z-s_{0}^{\varepsilon}\right]}$ for every $z<s_{0}^{\varepsilon}$.

Proof. Using expansion $\psi_{0}^{\varepsilon}=\psi_{0}+\varepsilon w_{0}+O\left(\varepsilon^{2}\right) e^{\Lambda(3 R) z}$ and $\lambda<\Lambda(2 R)<2 \lambda$ we can compute

$$
\begin{aligned}
\ln \frac{\psi_{0}^{\varepsilon^{\prime}}(z)}{\psi_{0}^{\varepsilon^{\prime}}\left(s_{0}^{\varepsilon}\right)}-\lambda\left[z-s_{0}^{\varepsilon}\right] & =\int_{s_{0}^{\varepsilon}}^{z}\left(\frac{\psi_{0}^{\varepsilon^{\prime \prime}}(x)}{\psi_{0}^{\varepsilon^{\prime}}(x)}-\lambda\right) d x \\
& \leqslant \frac{\varepsilon g^{2}(b)}{g^{\prime}(b)} \int_{z}^{s_{0}^{\varepsilon}}\left\{[\Lambda(2 R)+M \varepsilon][\Lambda(2 R)-\lambda] e^{[\Lambda(2 R)-\lambda][x-b]}\right. \\
& \left.-2 \lambda^{2} e^{\lambda[x-b]}\right\} d x<0,
\end{aligned}
$$

since there exists $\hat{x}<s_{0}^{\varepsilon}$ such that the integrand is positive in $(-\infty, \hat{x})$ and negative in $\left(\hat{x}, s_{0}^{\varepsilon}\right]$, so the integral attains its maximum either at $z=s_{0}^{\varepsilon}$ or $z=-\infty$. Hence, $\psi_{0}^{\varepsilon^{\prime}}(z) \leqslant \psi_{0}^{\varepsilon^{\prime}}\left(s_{0}^{\varepsilon}\right) e^{\lambda\left[z-s_{0}^{\varepsilon}\right]}=$ $g_{\varepsilon}^{\prime}\left(s_{0}^{\varepsilon}\right) e^{\lambda\left[z-s_{0}^{\varepsilon}\right]}=g_{\varepsilon}^{\prime}(z) e^{\left[\lambda-1_{\varepsilon}\right]\left[z-s_{0}^{\varepsilon}\right]}$.

Lemma 2.5 Let $M_{0}=\frac{2 g^{\prime}(b)}{R-1-\nu}=\frac{2 \lambda}{(\lambda-1)(R-1-v)}$. There exists a positive constant $M$ that depends only on $R$ and $v$ such that for every sufficiently small positive $\varepsilon$,

$$
0<-w_{0}^{\varepsilon}<\left[M_{0}+M \varepsilon\right]\left[g_{\varepsilon}^{\prime}-\psi_{0}^{\varepsilon{ }^{\prime}}\right] \text { in }\left(-\infty, s_{0}^{\varepsilon}\right) .
$$

Proof. Set $K=M_{0}+M \varepsilon$ and $\zeta=w_{0}^{\varepsilon}+K\left[g_{\varepsilon}^{\prime}-\psi_{0}^{\varepsilon^{\prime}}\right]$. Using $g_{\varepsilon}^{\prime \prime}=1_{\varepsilon} g_{\varepsilon}^{\prime}$ we have

$$
\begin{aligned}
-\zeta^{\prime \prime} & +\left(2 \varphi_{0}^{\varepsilon^{\prime}}-v\right) \zeta^{\prime}+R \zeta \\
& =\left\{-R \varepsilon v_{0}^{\varepsilon}-R w_{0}^{\varepsilon}-2 \psi_{0}^{\varepsilon^{\prime 2}}\right\}+K g_{\varepsilon}^{\prime}\left\{-1_{\varepsilon}^{2}+\left[2 \varphi_{0}^{\varepsilon^{\prime}}-v\right] 1_{\varepsilon}+R\right\}+K\left\{R \varepsilon w_{0}^{\varepsilon^{\prime}}+2 \varphi_{0}^{\varepsilon^{\prime \prime}} \psi_{0}^{\varepsilon^{\prime}}\right\} \\
& \geqslant-R \varepsilon v_{0}^{\varepsilon}-2 g_{\varepsilon}^{\prime 2}+K g_{\varepsilon}^{\prime}\left\{-1_{\varepsilon}^{2}-v 1_{\varepsilon}+R\right\}+K R \varepsilon w_{0}^{\varepsilon^{\prime}} \\
& \geqslant g_{\varepsilon}^{\prime}\left\{K\left[R-1_{\varepsilon}^{2}-v 1_{\varepsilon}\right]-2 g_{\varepsilon}^{\prime}\left(s_{0}^{\varepsilon}\right)-\varepsilon \frac{R v_{0}^{\varepsilon}-R K w_{0}^{\varepsilon^{\prime}}}{g_{\varepsilon}^{\prime}}\right\} \\
& =g_{\varepsilon}^{\prime}\left\{K[R-1-v]-2 g^{\prime}(b)+O(\varepsilon)[1+K]\right\}>0 .
\end{aligned}
$$

Since $\zeta\left(s_{0}^{\varepsilon}\right)=0$ and $\zeta(-\infty)=0$, it follows from the maximum principle that $\zeta>0$ in $\left(-\infty, s_{0}^{\varepsilon}\right)$. 
2.6 Modification of $\left(\varphi_{0}^{\varepsilon}, \psi_{0}^{\varepsilon}, w_{0}^{\varepsilon}, v_{0}^{\varepsilon}\right)$ in $\left[s_{0}^{\varepsilon}, \infty\right)$

For convenience, we redefine $\left(\varphi_{0}^{\varepsilon}, \psi_{0}^{\varepsilon}, w_{0}^{\varepsilon}, v_{0}^{\varepsilon}\right)$ in $\left[s_{0}^{\varepsilon}, \infty\right)$ as follows: for $z>s_{0}^{\varepsilon}$,

$$
\begin{aligned}
& \psi_{0}^{\varepsilon}(z)=g_{\varepsilon}(z), \quad w_{0}^{\varepsilon}(z)=0, v_{0}^{\varepsilon}(z)=0, \\
& \varphi_{0}^{\varepsilon}(z)=\varphi_{0}^{\varepsilon}\left(s_{0}^{\varepsilon}\right)+\varphi_{0}^{\varepsilon \prime}\left(s_{0}^{\varepsilon}\right)\left(z-s_{0}^{\varepsilon}\right)+\frac{1}{2} \varphi_{0}^{\varepsilon^{\prime \prime}}\left(s_{0}^{\varepsilon}\right)\left[z-s_{0}^{\varepsilon}\right]^{2} .
\end{aligned}
$$

Note that $\varphi_{0}^{\varepsilon^{\prime \prime}}\left(s_{0}^{\varepsilon}\right)=\varepsilon \psi_{0}^{\varepsilon^{\prime \prime}}\left(s_{0}^{\varepsilon}\right)+O\left(\varepsilon^{2}\right)>0$.

\section{Local existence of the approximated free boundary problem}

In this section, we establish the well-posedness of the following free boundary problem:

$$
\begin{cases}R a w_{a}^{\varepsilon}=w_{z z}^{\varepsilon}+\left(v-2 \varphi_{z}^{\varepsilon}\right) w_{z}^{\varepsilon}-2 R w^{\varepsilon}-2 \psi_{z}^{\varepsilon 2} & \forall a \in[\varepsilon, T), z<s_{\varepsilon}(a), \\ w^{\varepsilon}(z, a)=0 & \forall a \in[\varepsilon, T), z \geqslant s_{\varepsilon}(a), \\ \psi^{\varepsilon}(z, a)=\psi_{0}^{\varepsilon}(z)+\int_{\varepsilon}^{a} w^{\varepsilon}(z, t) d t & \forall a \in[\varepsilon, T), z \in \mathbb{R}, \\ \varphi^{\varepsilon}(z, a)=\varphi_{0}^{\varepsilon}(z)+\int_{\varepsilon}^{a} \psi^{\varepsilon}(z, t) d t & \forall a \in[\varepsilon, T), z \in \mathbb{R}, \\ l^{\varepsilon}(z, a)=R g_{\varepsilon}(z)+\left[2 \varphi_{z}^{\varepsilon}(z, a)-v\right] g_{\varepsilon}^{\prime}(z)-g_{\varepsilon}^{\prime \prime}(z) & \forall a \in[\varepsilon, T), z \in \mathbb{R} \\ S_{\varepsilon}(a)=s_{0}^{\varepsilon}-\int_{\varepsilon}^{a} \frac{w_{\varepsilon}^{\varepsilon}\left(s_{\varepsilon}(t), t\right)}{l^{\varepsilon}\left(s_{\varepsilon}(t), t\right)} d t & \forall a \in[\varepsilon, T), \\ w^{\varepsilon}(z, \varepsilon)=w_{0}^{\varepsilon}(z) \quad \forall z \in \mathbb{R} . & \end{cases}
$$

Since we shall show that $s_{\varepsilon}^{\prime}<0$, the initial values of $\left(\varphi_{0}^{\varepsilon}, \psi_{0}^{\varepsilon}, w_{0}^{\varepsilon}\right)$ in $\left(s_{0}^{\varepsilon}, \infty\right)$ don't affect the solution in the set $D:=\left\{(z, a) \mid a \in[\varepsilon, T), z \leqslant s_{\varepsilon}(a)\right\}$.

LEMma 3.1 (Local Existence) Let $s_{0}^{\varepsilon}$ be defined in Lemma 2.2, $\left(\varphi_{0}^{\varepsilon}, \psi_{0}^{\varepsilon}, w_{0}^{\varepsilon}\right)$ in $\left(-\infty, s_{0}^{\varepsilon}\right]$ be as in Lemma 2.1, and $g_{\varepsilon}$ be as in (2.3). Extend $\left(\varphi_{0}^{\varepsilon}, \psi_{0}^{\varepsilon}, w_{0}^{\varepsilon}\right)$ in $\left(s_{0}^{\varepsilon}, \infty\right)$ as in (2.4).

Then for every sufficiently small positive $\varepsilon$, there exists $T \in(\varepsilon, 1]$ such that problem (3.1) admits a unique classical solution $\left(\varphi^{\varepsilon}, \psi^{\varepsilon}, w^{\varepsilon}, s_{\varepsilon}\right)$. In addition, for every $a \in[\varepsilon, T), s_{\varepsilon}^{\prime}(a)<$ $0<l^{\varepsilon}\left(s_{\varepsilon}(a), a\right)$ and for every $\alpha \in(0,1), s_{\varepsilon} \in C^{2+\alpha / 2}([\varepsilon, T)) \cap C^{\infty}((\varepsilon, T))$ and $\varphi^{\varepsilon}, \psi^{\varepsilon}, w^{\varepsilon} \in$ $C^{3+\alpha,(3+\alpha) / 2}(D) \cap C^{\infty}\left(D \backslash\left(s_{0}^{\varepsilon}, \varepsilon\right)\right)$, where $D=\left\{(z, a) \mid a \in[\varepsilon, T), z \leqslant s_{\varepsilon}(a)\right\}$.

Proof. Using contraction mapping theorem, we divide the proof into 6 steps.

1. First we transform the free boundary problem to a fixed boundary problem.

Introduce $x=z-s_{\varepsilon}(a)$ and

$W(x, a)=w^{\varepsilon}\left(s_{\varepsilon}(a)+x, a\right), \quad \Psi(x, a)=\psi^{\varepsilon}\left(s_{\varepsilon}(a)+x, a\right), \quad \Phi(x, a)=\varphi^{\varepsilon}\left(x+s_{\varepsilon}(a), a\right)$.

Then problem (3.1) is equivalent to $W \equiv 0$ on $[0, \infty) \times[\varepsilon, T]$ and

$$
\begin{cases}R a W_{a}-W_{x x}-v W_{x}+2 R W=\left(R a s_{\varepsilon}^{\prime}-2 \Phi_{x}\right) W_{x}-2 \Psi_{x}^{2} & \forall a \in(\varepsilon, T], x<0, \\ \Psi(x, a)=\psi_{0}^{\varepsilon}\left(x+s_{\varepsilon}(a)\right)+\int_{\varepsilon}^{a} W\left(x+s_{\varepsilon}(a)-s_{\varepsilon}(t), t\right) d t & \forall a \in[\varepsilon, T], x \in \mathbb{R}, \\ \Phi(x, a)=\varphi_{0}^{\varepsilon}\left(x+s_{\varepsilon}(a)\right)+\int_{\varepsilon}^{a} \Psi\left(x+s_{\varepsilon}(a)-s_{\varepsilon}(t), t\right) d t & \forall a \in[\varepsilon, T], x \in \mathbb{R}, \\ s_{\varepsilon}(a)=s_{0}^{\varepsilon}-\int_{\varepsilon}^{a} \frac{w_{z}^{\varepsilon}\left(s_{\varepsilon}(t), t\right)}{L\left[\Phi, s_{\varepsilon}\right](t)} d t & \forall a \in[\varepsilon, T], \\ W(x, \varepsilon)=w_{0}^{\varepsilon}\left(x+s_{0}^{\varepsilon}\right) & \forall x \leqslant 0 .\end{cases}
$$


Here $L[\Phi, s]$ is a non-linear operator defined by

$$
L[\Phi, s](a)=R g_{\varepsilon}(s(a))+\left[2 \Phi_{x}(0, a)-v\right] g_{\varepsilon}^{\prime}(s(a))-g_{\varepsilon}^{\prime \prime}(s(a)) .
$$

2. Next we choose an appropriate function space.

Fix $T \in(\varepsilon, 1]$. Denote $I=[\varepsilon, T]$ and $Q=(-\infty, 0) \times(\varepsilon, T]$. Fix $\alpha=1 / 4$. We define

$$
\mathbf{X}:=\left\{\begin{array}{l|l}
(s, W) \mid \begin{array}{l}
s(\varepsilon)=s_{0}^{\varepsilon}, s^{\prime}(\varepsilon)=s_{1}^{\varepsilon},\left\|s^{\prime}-s_{1}^{\varepsilon}\right\|_{C^{\alpha}(I)} \leqslant \frac{1}{2}\left|s_{1}^{\varepsilon}\right|, \\
W(\cdot, \varepsilon)=w_{0}^{\varepsilon}\left(\cdot+s_{0}^{\varepsilon}\right), \quad\left\|W-W_{0}^{\varepsilon}\right\|_{C^{2+\alpha, 1+\alpha / 2}(\bar{Q})} \leqslant 1
\end{array}
\end{array}\right\},
$$

where $s_{1}^{\varepsilon}:=-v_{0}^{\varepsilon}\left(s_{0}^{\varepsilon}\right) / w_{0}^{\varepsilon^{\prime}}\left(s_{0}^{\varepsilon}\right)$ and

$$
W_{0}^{\varepsilon}(x, a)=w_{0}^{\varepsilon}\left(x+s_{0}^{\varepsilon}\right)+(a-\varepsilon)\left[v_{0}^{\varepsilon}\left(x+s_{0}^{\varepsilon}\right)+s_{1}^{\varepsilon} w_{0}^{\varepsilon^{\prime}}\left(x+s_{0}^{\varepsilon}\right)\right] .
$$

Fix $(s, W) \in \mathbf{X}$. Then $s^{\prime} \leqslant s_{1}^{\varepsilon}+\frac{1}{2}\left|s_{1}^{\varepsilon}\right|<0$ in $I$. We define, for $x \leqslant 0$ and $a \in I$,

$$
\begin{aligned}
& \Psi(x, a)=\psi_{0}^{\varepsilon}(x+s(a))+\int_{\varepsilon}^{a} W(x+s(a)-s(t), t) d t \\
& \Phi(x, a)=\varphi_{0}^{\varepsilon}(x+s(a))+(a-\varepsilon) \psi_{0}^{\varepsilon}(x+s(a))+\int_{\varepsilon}^{a}(a-t) W(x+s(a)-s(t), t) d t \\
& f(x, a)=\left[\operatorname{Ras}^{\prime}-2 \Phi_{x}\right] W_{x}-2 \Psi_{x}^{2} .
\end{aligned}
$$

Since $s^{\prime}<0$, these definitions do not depend on values of $W$ outside $\bar{Q}$. Also $W \in C^{2+\alpha, 1+\alpha / 2}(\bar{Q})$ implies $W_{x} \in C^{1+\alpha,(1+\alpha) / 2}(\bar{Q})$. As $s^{\prime} \in C^{\alpha}(I)$ and $1+\alpha>2 \alpha$, we have

$$
\|f\|_{C^{2 \alpha, \alpha}(\bar{Q})} \leqslant C_{\varepsilon}
$$

here and in the sequel, $C_{\varepsilon}$ denotes a generic constant that depends only on $\varepsilon, R$, and $\nu$.

3. We now define a map $(s, W) \in \mathbf{X} \rightarrow W^{\varepsilon}$.

We define $W^{\varepsilon}$ as the unique bounded solution of the linear initial boundary value problem

$$
\left\{\begin{array}{l}
R a W_{a}^{\varepsilon}-W_{x x}^{\varepsilon}-v W_{x}^{\varepsilon}+2 R W^{\varepsilon}=f \quad \text { in } Q, \\
W^{\varepsilon}(0, \cdot)=0, \quad W^{\varepsilon}(\cdot, \varepsilon)=w_{0}^{\varepsilon}\left(\cdot+s_{0}^{\varepsilon}\right) .
\end{array}\right.
$$

We show that the initial and the boundary values at the corner $(0, \varepsilon)$ satisfy the zeroth and first order compatibility conditions.

(i) Since $w_{0}^{\varepsilon}\left(s_{0}^{\varepsilon}\right)=0$, we see that $W^{\varepsilon}\left(0^{-}, \varepsilon\right)=w_{0}^{\varepsilon}\left(s_{0}^{\varepsilon}\right)=0=W^{\varepsilon}\left(0, \varepsilon^{+}\right)$.

(ii) The boundary value gives $W_{a}^{\varepsilon}\left(0, \varepsilon^{+}\right)=0$. The initial value and the differential equation give, for $x<0$,

$$
\begin{aligned}
W_{a}^{\varepsilon}(x, \varepsilon) & =\frac{1}{\varepsilon R}\left(f(x, \varepsilon)+w_{0}^{\varepsilon^{\prime \prime}}\left(x+s_{0}^{\varepsilon}\right)+v w_{0}^{\varepsilon^{\prime}}\left(x+s_{0}^{\varepsilon}\right)-2 R w_{0}^{\varepsilon}\left(x+s_{0}^{\varepsilon}\right)\right) \\
& =\frac{1}{\varepsilon R}\left(w_{0}^{\varepsilon^{\prime \prime}}+\left[v-2 \varphi_{0}^{\varepsilon^{\prime}}\right] w_{0}^{\varepsilon^{\prime}}-2 R w_{0}^{\varepsilon}-2 \psi_{0}^{\varepsilon^{\prime 2}}+\varepsilon R s_{1}^{\varepsilon} w_{0}^{\varepsilon^{\prime}}\right) \\
& =v_{0}^{\varepsilon}\left(x+s_{0}^{\varepsilon}\right)+s_{1}^{\varepsilon} w_{0}^{\varepsilon^{\prime}}\left(x+s_{0}^{\varepsilon}\right)=W_{0 a}^{\varepsilon}(x, \varepsilon),
\end{aligned}
$$

by the definition of $W_{0}^{\varepsilon}$. Since $s_{1}^{\varepsilon}:=-v_{0}^{\varepsilon}\left(s_{0}^{\varepsilon}\right) / w_{0}^{\varepsilon^{\prime}}\left(s_{0}^{\varepsilon}\right)$, we have $W_{a}^{\varepsilon}\left(0^{-}, \varepsilon\right)=0=W_{a}^{\varepsilon}\left(0, \varepsilon^{+}\right)$. 
Hence, there exists a unique bounded solution $W^{\varepsilon}$ and it satisfies

$$
\left\|W^{\varepsilon}\right\|_{C^{2+2 \alpha, 1+\alpha}(\bar{Q})} \leqslant C_{\varepsilon}\left\{\left\|w_{0}^{\varepsilon}\right\|_{C^{2+2 \alpha}\left(\left(-\infty, s_{0}^{\varepsilon}\right]\right)}+\|f\|_{C^{2 \alpha, \alpha}(\bar{Q})}\right\} \leqslant C_{\varepsilon} .
$$

In addition, since $W^{\varepsilon}(\cdot, \varepsilon)=W_{0}^{\varepsilon}(\cdot, \varepsilon)$ and $W_{a}^{\varepsilon}(\cdot, \varepsilon)=W_{0 a}^{\varepsilon}(\cdot, \varepsilon),\left\|W^{\varepsilon}-W_{0}^{\varepsilon}\right\|_{L^{\infty}}(Q) \leqslant C_{\varepsilon}(T-$ $\varepsilon)^{1+\alpha}$. Hence, extending $W^{\varepsilon}-W_{0}^{\varepsilon}$ by 0 for $a \leqslant \varepsilon$ we obtain by interpolation that

$$
\left\|W^{\varepsilon}-W_{0}^{\varepsilon}\right\|_{C^{2+\alpha, 1+\alpha / 2}(\bar{Q})} \leqslant C\left\|W^{\varepsilon}-W_{0}^{\varepsilon}\right\|_{C^{2+2 \alpha, 1+\alpha}(\bar{Q})}^{\frac{2+\alpha}{2+2 \alpha}}\left\|W^{\varepsilon}-W_{0}^{\varepsilon}\right\|_{L^{\infty}(Q)}^{\frac{\alpha}{2+2 \alpha}} \leqslant C_{\varepsilon}(T-\varepsilon)^{\frac{\alpha}{2}} .
$$

4. Next we define a map $(s, W) \in \mathbf{X} \rightarrow s_{\varepsilon}$.

By our construction of initial data, $L[\Phi, s](\varepsilon)=\psi_{0}^{\varepsilon \prime \prime}\left(s_{0}^{\varepsilon}\right)-g_{\varepsilon}^{\prime \prime}\left(s_{0}^{\varepsilon}\right)=-w_{0}^{\varepsilon^{\prime}}\left(s_{0}^{\varepsilon}\right) / s_{1}^{\varepsilon}>0$. Also, in $I$,

$$
(L[\Phi, s])^{\prime}=g_{\varepsilon}^{\prime}(s)\left[2 \Phi_{x a}(0, a)+\left\{R+\left[2 \Phi_{x}(0, a)-v\right] 1_{\varepsilon}-1_{\varepsilon}^{2}\right\} s^{\prime}\right] .
$$

Since $(s, W) \in \mathbf{X}$, we have $\Phi_{x a} \in C^{\alpha, \alpha / 2}(\bar{Q})$ so

$$
\|L[\Phi, s]\|_{C^{1+\alpha / 2}(I)} \leqslant C_{\varepsilon}
$$

Let $T_{1}:=\left[\psi_{0}^{\varepsilon \prime}\left(s_{0}^{\varepsilon}\right)-g_{\varepsilon}^{\prime \prime}\left(s_{0}^{\varepsilon}\right)\right] /\left(2 C_{\varepsilon}\right)$. Assume that $\varepsilon<T \leqslant \varepsilon+T_{1}$. Then

$$
L[\Phi, s](a) \geqslant L[\Phi, s](\varepsilon)-C_{\varepsilon}(T-\varepsilon)>\frac{1}{2}\left[\psi_{0}^{\varepsilon^{\prime \prime}}\left(s_{0}^{\varepsilon}\right)-g_{\varepsilon}^{\prime \prime}\left(s_{0}^{\varepsilon}\right)\right]>0 \quad \forall a \in I .
$$

Hence, we can define

$$
s_{\varepsilon}(a)=s_{0}^{\varepsilon}-\int_{\varepsilon}^{a} \frac{W_{x}^{\varepsilon}(0, t)}{L[\Phi, s](t)} d t \quad \forall a \in[\varepsilon, T] .
$$

It is easy to see that $s_{\varepsilon}(\varepsilon)=s_{0}^{\varepsilon}$ and $s_{\varepsilon}^{\prime}(\varepsilon)=s_{1}^{\varepsilon}$. Since $W_{x}^{\varepsilon} \in C^{1+2 \alpha, 1 / 2+\alpha}(\bar{Q})$, we have $\left\|s_{\varepsilon}^{\prime}\right\|_{C^{1 / 2+\alpha}(I)} \leqslant C_{\varepsilon}$ and $\left\|s_{\varepsilon}^{\prime}-s_{1}^{\varepsilon}\right\|_{C^{0}(I)} \leqslant C_{\varepsilon}(T-\varepsilon)^{1 / 2+\alpha}$. By interpolation,

$$
\left\|s_{\varepsilon}^{\prime}-s_{1}^{\varepsilon}\right\|_{C^{\alpha}(I)} \leqslant C\left\|s_{\varepsilon}^{\prime}-s_{1}^{\varepsilon}\right\|_{C^{1 / 2+\alpha}(I)}^{\frac{\alpha}{1 / 2+\alpha}}\left\|s_{\varepsilon}^{\prime}-s_{1}^{\varepsilon}\right\|_{C^{0}(I)}^{\frac{1 / 2}{1 / 2+\alpha}} \leqslant C_{\varepsilon}(T-\varepsilon)^{\frac{1}{2}} .
$$

Hence, if $0<T-\varepsilon \ll 1$, the map $(s, W) \rightarrow\left(s_{\varepsilon}, W^{\varepsilon}\right)$ is well-defined and maps $\mathbf{X}$ to itself.

5. Now we show that if $0<T-\varepsilon \ll 1$, the map $(s, W) \in \mathbf{X} \rightarrow\left(s_{\varepsilon}, W^{\varepsilon}\right)$ is a contraction.

Suppose for $i=1,2,\left(s_{i}, W_{i}\right) \in \mathbf{X}$. We denote by $\Phi_{i}, \Psi_{i}, f_{i}, W_{i}^{\varepsilon}, s_{\varepsilon}^{i}$ the corresponding functions defined in Steps 2-4.

We first estimate $f_{1}-f_{2}$. We decompose $\Psi_{1 x}-\Psi_{2 x}$ as $I_{1}+I_{2}+I_{3}$ where

$$
\begin{aligned}
& I_{1}=\psi_{0}^{\varepsilon^{\prime}}\left(x+s_{1}(a)\right)-\psi_{0}^{\varepsilon^{\prime}}\left(x+s_{2}(a)\right)=\int_{0}^{\left[s_{1}-s_{2}\right](a)} \psi_{0}^{\varepsilon^{\prime \prime}}\left(x+s_{2}(a)+y\right) d y, \\
& I_{2}=\int_{\varepsilon}^{a}\left[W_{1 x}-W_{2 x}\right]\left(x+s_{1}(a)-s_{1}(t), t\right) d t, \\
& I_{3}=\int_{\varepsilon}^{a} \int_{0}^{\left[s_{1}-s_{2}\right](a)-\left[s_{1}-s_{2}\right](t)} W_{2 x x}\left(x+s_{2}(a)-s_{2}(t)+y, t\right) d y d t .
\end{aligned}
$$


We find that

$$
\begin{aligned}
&\left\|I_{1}\right\|_{C^{\alpha}(\bar{Q})} \leqslant C\left\|s_{2}-s_{1}\right\|_{C^{\alpha}(I)} \leqslant C_{\varepsilon}(T-\varepsilon)^{1-\alpha}\left\|s_{1}^{\prime}-s_{2}^{\prime}\right\|_{C^{0}(I)}, \\
&\left\|I_{2}\right\|_{C^{\alpha}(\bar{Q})} \leqslant C(T-\varepsilon)^{1-\alpha}\left\|W_{1 x}-W_{2 x}\right\|_{C^{\alpha, 0}(\bar{Q})} \\
&\left\|I_{3}\right\|_{C^{\alpha}(\bar{Q})} \leqslant C(T-\varepsilon)^{1-\alpha}\left\|W_{2 x x}\right\|_{C^{\alpha, 0}(\bar{Q})}\left\|s_{1}-s_{2}\right\|_{C^{\alpha}(I)} .
\end{aligned}
$$

Hence,

$$
\left\|\Psi_{1 x}-\Psi_{2 x}\right\|_{C^{\alpha}(\bar{Q})} \leqslant C(T-\varepsilon)^{1-\alpha}\left(\left\|s_{1}^{\prime}-s_{2}^{\prime}\right\|_{C^{0}(I)}+\left\|W_{1 x}-W_{2 x}\right\|_{C^{\alpha, 0}(\bar{Q})}\right) .
$$

After a similar estimate for $\Phi_{1 x}-\Phi_{2 x}$ we then derive that

$$
\begin{aligned}
\left\|f_{1}-f_{2}\right\|_{C^{\alpha, \alpha / 2}(\bar{Q})} & \leqslant C_{\varepsilon}\left(\left\|s_{1}^{\prime}-s_{2}^{\prime}\right\|_{C^{\alpha / 2}(I)}+\left\|W_{1 x}-W_{2 x}\right\|_{C^{\alpha, \alpha / 2}(\bar{Q})}\right) \\
& \leqslant C_{\varepsilon}(T-\varepsilon)^{\alpha / 2}\left(\left\|s_{1}^{\prime}-s_{2}^{\prime}\right\|_{C^{\alpha}(I)}+\left\|W_{1 x}-W_{2 x}\right\|_{C^{2 \alpha, \alpha}(\bar{Q})}\right) .
\end{aligned}
$$

Similarly, using the boundedness of $\left\|W_{2 x x}\right\|_{C^{\alpha, 0}(\bar{Q})}$ we find that

$$
\left\|L\left[\Phi_{1}, s_{1}\right]-L\left[\Phi_{2}, s_{2}\right]\right\|_{C^{\alpha}(I)} \leqslant C(T-\varepsilon)^{1-\alpha}\left(\left\|s_{1}^{\prime}-s_{2}^{\prime}\right\|_{C^{0}(I)}+\left\|W_{1 x}-W_{2 x}\right\|_{C^{\alpha, 0}(\bar{Q})}\right) .
$$

Now, by a parabolic estimate for linear equation,

$$
\begin{aligned}
\left\|W_{1}^{\varepsilon}-W_{2}^{\varepsilon}\right\|_{C^{2+\alpha, 1+\alpha / 2}(\bar{Q})} & \leqslant C_{\varepsilon}\left\|f_{1}-f_{2}\right\|_{C^{\alpha, \alpha / 2}(\bar{Q})} \\
& \leqslant C_{\varepsilon}(T-\varepsilon)^{\alpha / 2}\left(\left\|s_{1}^{\prime}-s_{2}^{\prime}\right\|_{C^{\alpha}(I)}+\left\|W_{1 x}-W_{2 x}\right\|_{C^{2 \alpha, \alpha}(\bar{Q})}\right)
\end{aligned}
$$

and by the definition of $s_{\varepsilon}^{i}$,

$$
\begin{aligned}
\left\|s_{\varepsilon}^{1 \prime}-s_{\varepsilon}^{2 \prime}\right\|_{C^{\alpha}(I)} & \leqslant C_{\varepsilon}\left\{\left\|L\left[\Phi_{1}, s_{1}\right]-L\left[\Phi_{2}, s_{2}\right]\right\|_{C^{\alpha}(I)}+\left\|W_{1 x}^{\varepsilon}-W_{2 x}^{\varepsilon}\right\|_{C^{0, \alpha}(\bar{Q})}\right\} \\
& \leqslant C_{\varepsilon}(T-\varepsilon)^{\alpha / 2}\left\{\left\|s_{1}^{\prime}-s_{2}^{\prime}\right\|_{C^{\alpha}(I)}+\left\|W_{1 x}-W_{2 x}\right\|_{C^{2 \alpha, \alpha}(\bar{Q})}\right\} .
\end{aligned}
$$

It follows that

$$
\begin{aligned}
& \left\|s_{\varepsilon}^{1 \prime}-s_{\varepsilon}^{2 \prime}\right\|_{C^{\alpha}(I)}+\left\|W_{1}^{\varepsilon}-W_{2}^{\varepsilon}\right\|_{C^{2+\alpha, 1+\alpha / 2}(\bar{Q})} \\
& \quad \leqslant C_{\varepsilon}(T-\varepsilon)^{\alpha / 2}\left\{\left\|s_{1}^{\prime}-s_{2}^{\prime}\right\|_{C^{\alpha}(I)}+\left\|W_{1}-W_{2}\right\|_{C^{2+\alpha, 1+\alpha / 2}(\bar{Q})}\right\} .
\end{aligned}
$$

Thus, when $0<T-\varepsilon \ll 1$, the map $(s, W) \in \mathbf{X} \rightarrow\left(s_{\varepsilon}, W^{\varepsilon}\right)$ maps $\mathbf{X}$ to itself and is a contraction.

Hence, there exists $T \in(\varepsilon, 1]$ such that the map $(s, W) \in \mathbf{X} \rightarrow\left(s_{\varepsilon}, W^{\varepsilon}\right)$ admits a unique fixed point. The fixed point provides a solution of (3.2), and also a solution of (3.1).

6. Finally, we show the needed regularity by a bootstrap argument.

Suppose $W^{\varepsilon} \in C^{\alpha, \alpha / 2}((-\infty, 0] \times(\varepsilon, T])$ and $s_{\varepsilon} \in C^{(\alpha+1) / 2}((\varepsilon, T])$, where $\alpha>1$ is not an integer. Then $f \in C^{\alpha-1,(\alpha-1) / 2}((-\infty, 0] \times(\varepsilon, T])$. Since $W^{\varepsilon}(0, \cdot) \equiv 0$ and $W^{\varepsilon}(\cdot, \varepsilon) \in$ $C^{\infty}((-\infty, 0])$, we obtain $W^{\varepsilon} \in C^{\alpha+1,(\alpha+1) / 2}((-\infty, 0] \times[\varepsilon, T] \backslash(0, \varepsilon))$. This implies that $W_{x}^{\varepsilon} \in$ $C^{\alpha, \alpha / 2}((-\infty, 0] \times(\varepsilon, T])$ and, by the equation for $s_{\varepsilon}, s_{\varepsilon} \in C^{(\alpha+2) / 2}((\varepsilon, T])$.

This bootstrap argument shows that $W^{\varepsilon} \in C^{\infty}((-\infty, 0] \times[\varepsilon, T] \backslash(0, \varepsilon))$ and $s_{\varepsilon} \in C^{\infty}((\varepsilon, T])$. Also, using the compatibility condition we can show that $W^{\varepsilon} \in C^{3+\alpha,(3+\alpha) / 2}((-\infty, 0] \times[\varepsilon, T])$ and $s_{\varepsilon}^{\prime} \in C^{(2+\alpha) / 2}(I)$ for any $\alpha \in(0,1)$. This completes the proof of the lemma. 


\section{A priori estimates of the approximate solution}

In this section, we establish a priori estimates that do not depend on $\varepsilon$ and $T$. In the sequel, $\left(\varphi^{\varepsilon}, \psi^{\varepsilon}, w^{\varepsilon}, s_{\varepsilon}\right)$ is the unique solution of (3.1) with $T \in(\varepsilon, \infty]$ and $0<\varepsilon \ll 1$. We assume that the solution satisfies $\ell^{\varepsilon}(a):=l^{\varepsilon}\left(s_{\varepsilon}(a), a\right)>0>s_{\varepsilon}^{\prime}(a)$ for every $a \in[\varepsilon, T)$.

\subsection{Differential equations and free boundary conditions}

LEMMA 4.1 Let $\left(\varphi^{\varepsilon}, \psi^{\varepsilon}, w^{\varepsilon}, s_{\varepsilon}\right)$ be a solution of (3.1) where $\ell^{\varepsilon}(a):=l^{\varepsilon}\left(s_{\varepsilon}(a), a\right)>0>s_{\varepsilon}^{\prime}(a)$ for each $a \in[\varepsilon, T)$. Set $\mathbf{N}^{\varepsilon}:=\left\{(z, a) \mid a \in[\varepsilon, T), z<s_{\varepsilon}(a)\right\}, \Gamma^{\varepsilon}:=\left\{(z, a) \mid a \in(\varepsilon, T), z=s_{\varepsilon}(a)\right\}$ and $v^{\varepsilon}=w_{a}^{\varepsilon}$. Then in $\mathbf{N}^{\varepsilon}$,

$$
\begin{aligned}
Q\left[\varphi^{\varepsilon}\right] & :=\operatorname{Ra}_{a}^{\varepsilon}-\varphi_{z z}^{\varepsilon}-v \varphi_{z}^{\varepsilon}+\varphi_{z}^{\varepsilon 2}=0, \\
\mathcal{L}_{1}\left[\psi^{\varepsilon}\right] & :=\operatorname{Ra} \psi_{a}^{\varepsilon}-\psi_{z z}^{\varepsilon}-v \psi_{z}^{\varepsilon}+2 \varphi_{z}^{\varepsilon} \psi_{z}^{\varepsilon}+R \psi^{\varepsilon}=0, \\
\mathcal{L}_{2}\left[w^{\varepsilon}\right] & :=\operatorname{Ra}_{a}^{\varepsilon}-w_{z z}^{\varepsilon}-v w_{z}^{\varepsilon}+2 \varphi_{z}^{\varepsilon} w_{z}^{\varepsilon}+2 R w^{\varepsilon}=-2 \psi_{z}^{\varepsilon 2}, \\
\mathcal{L}_{3}\left[v^{\varepsilon}\right] & :=\operatorname{Rav}_{a}^{\varepsilon}-v_{z z}^{\varepsilon}-v v_{z}^{\varepsilon}+2 \varphi_{z}^{\varepsilon} v_{z}^{\varepsilon}+3 R v^{\varepsilon}=-6 \psi_{z}^{\varepsilon} w_{z}^{\varepsilon} .
\end{aligned}
$$

In addition, $\left.\left(\varphi^{\varepsilon}, \psi^{\varepsilon}, w^{\varepsilon}, v^{\varepsilon}\right)\right|_{a=\varepsilon}=\left(\varphi_{0}^{\varepsilon}, \psi_{0}^{\varepsilon}, w_{0}^{\varepsilon}, v_{0}^{\varepsilon}\right)$ and

$$
\psi^{\varepsilon}=g_{\varepsilon}, \quad \psi_{z}^{\varepsilon}=g_{\varepsilon}^{\prime}, \quad w^{\varepsilon}=0, \quad \ell^{\varepsilon} s_{\varepsilon}^{\prime}=-w_{z}^{\varepsilon}, \quad v^{\varepsilon}=\ell^{\varepsilon} s_{\varepsilon}^{\prime 2} \quad \text { on } \boldsymbol{\Gamma}^{\varepsilon} .
$$

Consequently, denoting by $\llbracket f \rrbracket^{\varepsilon}$ the jump of $f$ across $\Gamma^{\varepsilon}$ we have

$$
\begin{gathered}
\llbracket \varphi^{\varepsilon} \rrbracket^{\varepsilon}=0, \quad \llbracket \varphi_{z}^{\varepsilon} \rrbracket^{\varepsilon}=0, \quad \llbracket \varphi_{z z}^{\varepsilon} \rrbracket^{\varepsilon}=0, \quad \llbracket \psi^{\varepsilon} \rrbracket^{\varepsilon}=0, \quad \llbracket \psi_{z}^{\varepsilon} \rrbracket^{\varepsilon}=0, \quad \llbracket w^{\varepsilon} \rrbracket^{\varepsilon}=0, \\
\llbracket \varphi_{z z z}^{\varepsilon} \rrbracket^{\varepsilon}=\ell^{\varepsilon} / s_{\varepsilon}^{\prime}, \quad \llbracket \psi_{z z}^{\varepsilon} \rrbracket^{\varepsilon}=-\ell^{\varepsilon}, \quad \llbracket w_{z}^{\varepsilon} \rrbracket^{\varepsilon}=\ell^{\varepsilon} s_{\varepsilon}^{\prime}, \quad \llbracket v^{\varepsilon} \rrbracket^{\varepsilon}=-\ell^{\varepsilon} s_{\varepsilon}^{\prime 2} .
\end{gathered}
$$

Proof. Note that $\left.\left(\varphi^{\varepsilon}, \psi^{\varepsilon}, w^{\varepsilon}\right)\right|_{a=\varepsilon}=\left(\varphi_{0}^{\varepsilon}, \psi_{0}^{\varepsilon}, w_{0}^{\varepsilon}\right)$. By the definition of $\left(\varphi_{0}^{\varepsilon}, \psi_{0}^{\varepsilon}, w_{0}^{\varepsilon}\right)$ we find that $Q\left[\varphi^{\varepsilon}\right](\cdot, \varepsilon)=0$ and $\mathcal{L}_{1}\left[\psi^{\varepsilon}\right](\cdot, \varepsilon)=0$ in $\left(-\infty, s_{0}^{\varepsilon}\right]$. Also, the differential equation in (3.1) for $w^{\varepsilon}$ can be written as

$$
0=\mathcal{L}_{2}\left[w^{\varepsilon}\right]+2 \psi_{z}^{\varepsilon 2}=\frac{\partial}{\partial a} \mathcal{L}_{1}\left[\psi^{\varepsilon}\right]=\frac{\partial^{2}}{\partial a^{2}} Q\left[\varphi^{\varepsilon}\right] \text { in } \mathbf{N}^{\varepsilon} .
$$

For each $z<s_{\varepsilon}(a)$, we have $z<s_{\varepsilon}(t)$ for $t \in[\varepsilon, a]$ since $s_{\varepsilon}^{\prime}<0$. Hence, integrating the equation over $[\varepsilon, a]$ we obtain $\mathcal{L}_{1}\left[\psi^{\varepsilon}\right]=0$ and $Q\left[\varphi^{\varepsilon}\right]=0$ in $\mathbf{N}^{\varepsilon}$. Differentiating $\mathcal{L}_{2}\left[w^{\varepsilon}\right]+2 \psi_{z}^{\varepsilon}=0$ we obtain $\mathcal{L}_{3}\left[v^{\varepsilon}\right]+6 \psi_{z}^{\varepsilon} w_{z}^{\varepsilon}=0$ in $\mathbf{N}^{\varepsilon}$. Setting $a=\varepsilon$ in $\mathcal{L}_{2}\left[w^{\varepsilon}\right]+2 \psi_{z}^{\varepsilon}=0$ and using the definition of $v_{0}^{\varepsilon}$ we obtain $v^{\varepsilon}(\cdot, \varepsilon)=v_{0}^{\varepsilon}$.

Next, set $J_{1}(a)=\psi^{\varepsilon}\left(s_{\varepsilon}(a), a\right)-g_{\varepsilon}\left(s_{\varepsilon}(a)\right)$ and $J_{2}(a)=\psi_{z}^{\varepsilon}\left(s_{\varepsilon}(a), a\right)-g_{\varepsilon}^{\prime}\left(s_{\varepsilon}(a)\right)$. We calculate

$$
\begin{aligned}
J_{1}^{\prime}(a) & =\left[\psi_{z}^{\varepsilon}-g_{\varepsilon}^{\prime}\right] s_{\varepsilon}^{\prime}+w^{\varepsilon}=s_{\varepsilon}^{\prime} J_{2}(a) \\
J_{2}^{\prime}(a) & =\left[\psi_{z z}^{\varepsilon}-g_{\varepsilon}^{\prime \prime}\right] s_{\varepsilon}^{\prime}+w_{z}^{\varepsilon} \\
& =\left[\psi_{z z}^{\varepsilon}-g_{\varepsilon}^{\prime \prime}\right] s_{\varepsilon}^{\prime}+\left[g_{\varepsilon}^{\prime \prime}+\left(v-2 \varphi_{z}^{\varepsilon}\right) g_{\varepsilon}^{\prime}-R g_{\varepsilon}\right] s_{\varepsilon}^{\prime} \\
& =R s_{\varepsilon}^{\prime}\left[\psi^{\varepsilon}-g_{\varepsilon}\right]-\left(v-2 \varphi_{z}^{\varepsilon}\right) s_{\varepsilon}^{\prime}\left[\psi_{z}^{\varepsilon}-g_{\varepsilon}^{\prime}\right]+s_{\varepsilon}^{\prime}\left[\psi_{z z}^{\varepsilon}+\left(v-2 \varphi_{z}^{\varepsilon}\right) \psi_{z}^{\varepsilon}-R \psi^{\varepsilon}\right] \\
& =R s_{\varepsilon}^{\prime} J_{1}(a)-\left(v-2 \varphi_{z}^{\varepsilon}\right) s_{\varepsilon}^{\prime} J_{2}(a)
\end{aligned}
$$

here we have used $w_{z}^{\varepsilon}=\left[g_{\varepsilon}^{\prime \prime}+\left(v-2 \varphi_{z}^{\varepsilon}\right) g_{\varepsilon}^{\prime}-R g_{\varepsilon}\right] s_{\varepsilon}^{\prime}$ and $\psi_{z z}^{\varepsilon}+\left(v-2 \varphi_{z}^{\varepsilon}\right) \psi_{z}^{\varepsilon}-R \psi^{\varepsilon}=R a \psi_{a}^{\varepsilon}=$ $R a w^{\varepsilon}=0$ on $\Gamma^{\varepsilon}$. Initially, $J_{1}(\varepsilon)=\psi_{0}^{\varepsilon}\left(s_{0}^{\varepsilon}\right)-g_{\varepsilon}\left(s_{0}^{\varepsilon}\right)=0$ and $J_{2}(\varepsilon)=\psi_{0}^{\varepsilon}\left(s_{0}^{\varepsilon}\right)-g_{\varepsilon}^{\prime}\left(s_{0}^{\varepsilon}\right)=0$. The 
uniqueness of the solution of the ode system for $\left(J_{1}, J_{2}\right)$ implies that $J_{1} \equiv 0$ and $J_{2} \equiv 0$. Thus, $\psi^{\varepsilon}=g_{\varepsilon}$ and $\psi_{z}^{\varepsilon}=g_{\varepsilon}^{\prime}$ on $\Gamma^{\varepsilon}$. Consequently, by the equation for $\psi^{\varepsilon}$,

$$
\begin{aligned}
\ell^{\varepsilon}(a) & :=R g_{\varepsilon}+\left(2 \varphi_{z}^{\varepsilon}-v\right) g_{\varepsilon}^{\prime}-\left.g_{\varepsilon}^{\prime \prime}\right|_{z=s_{\varepsilon}(a)} \\
& =R \psi^{\varepsilon}+\left(2 \varphi_{z}^{\varepsilon}-v\right) \psi_{z}^{\varepsilon}-\left.g_{\varepsilon}^{\prime \prime}\right|_{z=s_{\varepsilon}(a)}=\psi_{z z}^{\varepsilon}\left(s_{\varepsilon}(a), a\right)-g_{\varepsilon}^{\prime \prime}\left(s_{\varepsilon}(a)\right) .
\end{aligned}
$$

Differentiating $w^{\varepsilon}\left(s_{\varepsilon}(a), a\right)=0$ with respect to $a$ we obtain $v^{\varepsilon}=-w_{z}^{\varepsilon} s_{\varepsilon}^{\prime}=\ell^{\varepsilon} s_{\varepsilon}^{\prime 2}$ on $\Gamma^{\varepsilon}$.

We know $w^{\varepsilon} \equiv 0$ and $v^{\varepsilon} \equiv 0$ in $\mathbf{T}^{\varepsilon}:=\left\{(z, a) \mid a \in(\varepsilon, T), z>s_{\varepsilon}(a)\right\}$. Since $\psi^{\varepsilon}=g_{\varepsilon}$ and $\psi_{z}^{\varepsilon}=g_{\varepsilon}^{\prime}$ on $\boldsymbol{\Gamma}^{\varepsilon}$ and $s_{\varepsilon}^{\prime}<0$, by integrating $\psi_{a}^{\varepsilon}=w^{\varepsilon}$ we obtain $\psi^{\varepsilon} \equiv g_{\varepsilon}$ and $\psi_{z}^{\varepsilon} \equiv g_{\varepsilon}^{\prime}$ on $\overline{\mathbf{T}}^{\varepsilon}$. This implies that $\psi^{\varepsilon}$ and $\psi_{z}^{\varepsilon}$ are continuous on $\mathbb{R} \times[\varepsilon, T)$. It also implies that $\varphi^{\varepsilon}$ and $\varphi_{z}^{\varepsilon}$ are continuous on $\mathbb{R} \times[\varepsilon, T)$. In addition, $\llbracket w^{\varepsilon} \rrbracket^{\varepsilon}=0, \llbracket \psi_{z z}^{\varepsilon} \rrbracket^{\varepsilon}=-\ell^{\varepsilon}$, and $\llbracket w_{z}^{\varepsilon} \rrbracket^{\varepsilon}=\ell^{\varepsilon} s_{\varepsilon}^{\prime}$. Now differentiating $\llbracket \varphi_{z}^{\varepsilon} \rrbracket^{\varepsilon}=0$ with respect to $a$, we find $\llbracket \varphi_{z z}^{\varepsilon} \rrbracket^{\varepsilon} s_{\varepsilon}^{\prime}+\llbracket \psi_{z} \rrbracket^{\varepsilon}=0$, so $\llbracket \varphi_{z z}^{\varepsilon} \rrbracket^{\varepsilon}=0$. Differentiating $\llbracket \varphi_{z z}^{\varepsilon} \rrbracket^{\varepsilon}=0$ we obtain $\llbracket \varphi_{z z z}^{\varepsilon} \rrbracket^{\varepsilon} s_{\varepsilon}^{\prime}+\llbracket \psi_{z z} \rrbracket^{\varepsilon}=0$, so $\llbracket \varphi_{z z z}^{\varepsilon} \rrbracket^{\varepsilon}=-\llbracket \psi_{z z} \rrbracket^{\varepsilon} / s_{\varepsilon}^{\prime}=\ell^{\varepsilon} / s_{\varepsilon}^{\prime}$. Finally, differentiating $\llbracket w^{\varepsilon} \rrbracket=0$ we obtain $\llbracket w_{z}^{\varepsilon} \rrbracket^{\varepsilon} s_{\varepsilon}^{\prime}+\llbracket v^{\varepsilon} \rrbracket^{\varepsilon}=0$ so $\llbracket v^{\varepsilon} \rrbracket^{\varepsilon}=-s_{\varepsilon}^{\prime} \llbracket w_{z}^{\varepsilon} \rrbracket^{\varepsilon}=-\ell^{\varepsilon} s_{\varepsilon}^{\prime 2}$. This completes the proof.

REMARK 4.1 The formula $\left(\llbracket f \rrbracket^{\varepsilon}\right)^{\prime}=\llbracket f_{z} \rrbracket^{\varepsilon} s_{\varepsilon}^{\prime}+\llbracket f_{a} \rrbracket^{\varepsilon}$ is derived as follows. Suppose $f^{ \pm}$are $C^{1}$ functions in $\mathbb{R} \times(\varepsilon, T)$. Define $f=f^{+}$for $z>s_{\varepsilon}(a)$ and $f=f^{-}$for $z \leqslant s_{\varepsilon}(a)$. Then by definition, $\llbracket f \rrbracket^{\varepsilon}=f^{+}\left(s_{\varepsilon}(a), a\right)-f^{-}\left(s_{\varepsilon}(a), a\right)$ and

$$
\begin{aligned}
\left(\llbracket f \rrbracket^{\varepsilon}\right)^{\prime} & =\left\{f_{z}^{+}\left(s_{\varepsilon}(a), a\right)-f_{z}^{-}\left(s_{\varepsilon}(a), a\right)\right\} s_{\varepsilon}^{\prime}(a)+\left\{f_{a}^{+}\left(s_{\varepsilon}(a), a\right)-f_{a}^{-}\left(s_{\varepsilon}(a), a\right)\right\} \\
& =\llbracket f_{z} \rrbracket^{\varepsilon} s_{\varepsilon}^{\prime}+\llbracket f_{a} \rrbracket^{\varepsilon} .
\end{aligned}
$$

We use the default $\psi^{\varepsilon+} \equiv g_{\varepsilon}, w^{\varepsilon+} \equiv 0$, and $v^{\varepsilon+} \equiv 0$. However, there may not exist a smooth extension of $\varphi^{\varepsilon}$. The best regularity of an extension $\varphi^{\varepsilon-}$ of $\varphi^{\varepsilon}$ from $\mathbf{T}^{\varepsilon}$ to $\mathbb{R} \times[\varepsilon, T)$ depends on the compatibility of the boundary value $\varphi^{\varepsilon}\left(s_{\varepsilon}(a), a\right)$ at $a=\varepsilon$ and the initial value $\varphi_{0}^{\varepsilon}(z)$ at $z=s_{0}^{\varepsilon}$.

\subsection{Monotonicity and concavity}

Lemma 4.2 In $\mathbf{N}^{\varepsilon}, \psi^{\varepsilon}>0>w^{\varepsilon}$.

Consequently, denoting by $0^{\varepsilon}$ the root of $g_{\varepsilon}(\cdot)=0$ we have $0^{\varepsilon}<s_{\varepsilon}(a)<s_{0}^{\varepsilon}$ for all $a \in(\varepsilon, T)$.

Proof. The assertion $w^{\varepsilon}<0$ in $\mathbf{N}^{\varepsilon}$ follows from the maximum principle, since $\mathcal{L}_{2}\left[w^{\varepsilon}\right] \leqslant 0$ in $\mathbf{N}^{\varepsilon}$ and $w^{\varepsilon} \leqslant 0$ on the parabolic boundary of $\mathbf{N}^{\varepsilon}$. The assertion $\psi^{\varepsilon}>0$ in $\mathbf{N}^{\varepsilon}$ also follows from the maximum principle since $\mathcal{L}_{1}\left[\psi^{\varepsilon}\right]=0$ in $\mathbf{N}^{\varepsilon}, \psi^{\varepsilon}(\cdot, \varepsilon)>0$ and $\psi_{z}^{\varepsilon}=g_{\varepsilon}^{\prime}>0$ on $\boldsymbol{\Gamma}^{\varepsilon}$. Finally $g_{\varepsilon}\left(s_{\varepsilon}(a)\right)=\psi^{\varepsilon}\left(s_{\varepsilon}(a), a\right)>0$ implies that $s_{\varepsilon}(a)>0^{\varepsilon}$ for all $a \in[\varepsilon, T)$.

Lemma 4.3 In $\mathbb{R} \times[\varepsilon, T), \varphi_{z z}^{\varepsilon}>0$; on $\overline{\mathbf{N}}^{\varepsilon}, 0<\psi_{z}^{\varepsilon} \leqslant g_{\varepsilon}^{\prime} e^{\left[\lambda-1_{\varepsilon}\right]\left[z-s_{\varepsilon}(a)\right]}$ and $\varphi_{z z z}^{\varepsilon}>0$.

Proof. 1. First we show that $\varphi_{z z}^{\varepsilon}>0$ in $\mathbb{R} \times[\varepsilon, T)$.

Set $\zeta=\varphi_{z z}^{\varepsilon}$. Differentiating $Q\left[\varphi^{\varepsilon}\right]=0$ with respect to $z$ twice we obtain $R a \zeta_{a}-\zeta_{z z}-(v-$ $\left.2 \varphi_{z}^{\varepsilon}\right) \zeta_{z}+2 \varphi_{z z}^{\varepsilon} \zeta=0$ in $\mathbf{N}^{\varepsilon}$. Initially, $\zeta(\cdot, \varepsilon)=\varphi_{0}^{\varepsilon^{\prime \prime}}=\varepsilon \psi_{0}^{\varepsilon \prime \prime}+O\left(\varepsilon^{2}\right) e^{\Lambda(2 R) z}=\left[\varepsilon+O\left(\varepsilon^{2}\right)\right] \psi_{0}^{\varepsilon^{\prime \prime}}>0$ (assuming $0<\varepsilon \ll 1$ ). Also, differentiating $Q\left[\varphi^{\varepsilon}\right]=0$ with respect to $z$ and set $z=s_{\varepsilon}(a)$ we obtain

$$
\zeta_{z}+\left.\left(v-2 \varphi_{z}^{\varepsilon}\right) \zeta\right|_{z=s_{\varepsilon}(a)}=\operatorname{Ra}_{z}^{\varepsilon}=\operatorname{Rag}_{\varepsilon}^{\prime}\left(s_{\varepsilon}(a)\right)>0
$$

Hence, by the maximum principle, $\zeta=\varphi_{z z}^{\varepsilon}>0$ on $\overline{\mathbf{N}}^{\varepsilon}$. 
Next, for $z \in\left(s_{\varepsilon}(a), s_{0}^{\varepsilon}\right]$, denoting by $a=a_{\varepsilon}(z)$ the inverse function of $z=s_{\varepsilon}(a)$ we have

$$
\begin{aligned}
\varphi^{\varepsilon}(z, a) & =\varphi^{\varepsilon}\left(z, a_{\varepsilon}(z)\right)+\left[a-a_{\varepsilon}(z)\right] g(z), \\
\varphi_{z}^{\varepsilon}(z, a) & =\varphi_{z}^{\varepsilon}\left(z, a_{\varepsilon}(z)\right)+\left[a-a_{\varepsilon}(z)\right] g_{\varepsilon}^{\prime}(z), \\
\varphi_{z z}^{\varepsilon}(z, a) & =\varphi_{z z}^{\varepsilon}\left(z, a_{\varepsilon}(z)\right)+\left[a-a_{\varepsilon}(z)\right] g_{\varepsilon}^{\prime \prime}(z)>0,
\end{aligned}
$$

since $\llbracket \psi \rrbracket^{\varepsilon}=\llbracket \psi_{z}^{\varepsilon} \rrbracket^{\varepsilon}=0$. Hence, $\varphi_{z z}^{\varepsilon} \in C\left(\left(-\infty, s_{0}^{\varepsilon}\right] \times[\varepsilon, T)\right)$ and $\varphi_{z z}^{\varepsilon}>0$ in $\left(-\infty, s_{0}^{\varepsilon}\right] \times[\varepsilon, T)$.

Finally, since $\varphi^{\varepsilon}=\varphi_{0}^{\varepsilon}+[a-\varepsilon] g_{\varepsilon}$ for $z>s_{0}^{\varepsilon}$, we see that $\varphi_{z z}^{\varepsilon} \in C(\mathbb{R} \times[\varepsilon, T))$ and $\varphi_{z z}^{\varepsilon}>0$.

2. Next we estimate $\psi_{z}^{\varepsilon}$, which satisfies $\left(\mathcal{L}_{1}+2 \varphi_{z z}^{\varepsilon}\right) \psi_{z}^{\varepsilon}=0$ in $\mathbf{N}^{\varepsilon}$.

As $\psi_{z}^{\varepsilon}>0$ on the parabolic boundary of $\mathbf{N}^{\varepsilon}$, we have $\psi_{z}^{\varepsilon}>0$ on $\overline{\mathbf{N}}^{\varepsilon}$.

By Lemma 2.4, we see that $\psi_{z}^{\varepsilon} \leqslant \zeta:=g_{\varepsilon}^{\prime} e^{\left[\lambda-1_{\varepsilon}\right]\left[z-s_{\varepsilon}(a)\right]}$ on the parabolic boundary of $\mathbf{N}^{\varepsilon}$. Also using $g_{\varepsilon}^{\prime}(z)=g_{\varepsilon}^{\prime}(0) e^{1_{\varepsilon} z}$ we have $\zeta=g_{\varepsilon}^{\prime}(0) e^{\lambda z-\left[\lambda-1_{\varepsilon}\right] s_{\varepsilon}(a)}$. Hence,

$$
\left.\left(\mathcal{L}_{1}+2 \varphi_{z z}^{\varepsilon}\right)\right\}=\zeta\left\{-\left[\lambda-1_{\varepsilon}\right] \operatorname{Ras}_{\varepsilon}^{\prime}-\lambda^{2}-\nu \lambda+2 \lambda \varphi_{z}^{\varepsilon}+2 \varphi_{z z}^{\varepsilon}+R\right\}>0,
$$

since $s_{\varepsilon}^{\prime}<0, \lambda^{2}+\nu \lambda-R=0, \varphi_{z}^{\varepsilon}>0$, and $\varphi_{z z}^{\varepsilon}>0$. It then follows from comparison principle that $\psi_{z}^{\varepsilon}<\zeta=g_{\varepsilon}^{\prime} e^{\left[\lambda-1_{\varepsilon}\right]\left[z-s_{\varepsilon}(a)\right]}$ in $\mathbf{N}^{\varepsilon}$.

3. Finally we show that $\varphi_{z z z}^{\varepsilon}>0$ on $\overline{\mathbf{N}}^{\varepsilon}$.

Set $\zeta=\varphi_{z z z}^{\varepsilon}$. Then $R a \zeta_{a}-\zeta_{z z}-v \zeta_{z}+2 \varphi_{z}^{\varepsilon} \zeta_{z}+6 \varphi_{z z}^{\varepsilon} \zeta=0$ in $\mathbf{N}^{\varepsilon}$. Initially, $\zeta(\cdot, \varepsilon)=$ $\left[\varepsilon+O\left(\varepsilon^{2}\right)\right] \psi_{0}^{\prime \prime \prime}>0$. On $\Gamma^{\varepsilon}$, differentiating $Q\left[\varphi^{\varepsilon}\right]=0$ with respect to $z$ twice we have

$$
\zeta_{z}+\left(v-2 \varphi_{z}^{\varepsilon}\right) \zeta=\operatorname{Ra} \psi_{z z}^{\varepsilon}+2 \varphi_{z z}^{\varepsilon}{ }^{2}>\operatorname{Rag}_{\varepsilon}^{\prime \prime}>0 \text { on } \Gamma^{\varepsilon} \text {. }
$$

It then follows from the maximum principle that $\varphi_{z z z}^{\varepsilon}>0$ on $\overline{\mathbf{N}}^{\varepsilon}$.

\subsection{Jump of $\varphi_{z z z}^{\varepsilon}$}

LEMMA 4.4 (1) For $a \in(\varepsilon, T)$ and $z \in \mathbb{R}$,

$$
\varphi_{z z z}^{\varepsilon}(z, a)=\varphi_{0}^{\varepsilon^{\prime \prime \prime}}(z)+\frac{\ell^{\varepsilon}\left(a_{\varepsilon}(z)\right)}{s_{\varepsilon}^{\prime}\left(a_{\varepsilon}(z)\right)} \mathbf{1}_{\left\{s_{\varepsilon}(a)<z \leqslant s_{0}^{\varepsilon}\right\}}+(a-\varepsilon) g_{\varepsilon}^{\prime \prime \prime}(z)+o(1)
$$

where $a_{\varepsilon}(z)$ is the inverse of $s_{\varepsilon}(z)$ with extension $a_{\varepsilon}(z)=\varepsilon$ for $z>s_{0}^{\varepsilon}$ and $a_{\varepsilon}(z)=T$ for $z \leqslant s_{\varepsilon}(T)$ and $\lim _{a \searrow \varepsilon}\|o(1)\|_{L^{\infty}(\mathbb{R})}=0$.

(2) For each $a \in(\varepsilon, T)$,

$$
\lim _{h \searrow 0}\left[\varphi_{z z z}^{\varepsilon}\left(s_{0}^{\varepsilon}+h, a\right)-\varphi_{z z z}^{\varepsilon}\left(s_{0}^{\varepsilon}-h, a\right)\right]=O(\varepsilon)-\frac{\ell^{\varepsilon}(\varepsilon)}{s_{\varepsilon}^{\prime}(\varepsilon)}=\frac{\lambda^{2}(\lambda-1)^{2}}{2 \lambda-\Lambda(2 R)}+O(\varepsilon) .
$$

Proof. (i) When $z>s_{0}^{\varepsilon}$, we have $\varphi_{z z z}^{\varepsilon}=\varphi_{0}^{\varepsilon^{\prime \prime \prime}}(z)+[a-\varepsilon] g_{\varepsilon}^{\prime \prime \prime}(z)$.

(ii) When $z \leqslant s_{\varepsilon}(a)$,

$$
\varphi_{z z z}^{\varepsilon}(z, a)=\varphi_{0}^{\varepsilon^{\prime \prime \prime}}(z)+\int_{\varepsilon}^{a} \psi_{z z z}^{\varepsilon}(z, t) d t
$$


(iii) When $z \in\left(s_{\varepsilon}(a), s_{0}^{\varepsilon}\right]$, we have

$$
\begin{aligned}
\varphi_{z z z}^{\varepsilon}(z, a) & =\varphi_{z z z}^{\varepsilon}\left(z, a_{\varepsilon}(z)\right)+\left(a-a_{\varepsilon}(z)\right) g_{\varepsilon}^{\prime \prime \prime}(z)+\left[\psi_{z z}^{\varepsilon}\left(z, a_{\varepsilon}(z)\right)-g_{\varepsilon}^{\prime \prime}(z)\right] a_{\varepsilon}^{\prime}(z) \\
& =\varphi_{0}^{\varepsilon^{\prime \prime \prime}}(z)+\int_{\varepsilon}^{a_{\varepsilon}(z)} \psi_{z z z}^{\varepsilon}(z, t) d t+\left(a-a_{\varepsilon}(z)\right) g_{\varepsilon}^{\prime \prime \prime}(z)+\frac{\ell^{\varepsilon}\left(a_{\varepsilon}(a)\right)}{s_{\varepsilon}^{\prime}\left(a_{\varepsilon}(z)\right)}
\end{aligned}
$$

Combining (i), (ii), and (iii) we have

$$
\begin{aligned}
\varphi_{z z z}^{\varepsilon}(z, a)-\left\{\varphi_{0}^{\varepsilon^{\prime \prime \prime}}(z)+\right. & \left.\frac{\ell^{\varepsilon}\left(a_{\varepsilon}(z)\right)}{s_{\varepsilon}^{\prime}\left(a_{\varepsilon}(z)\right)} \mathbf{1}_{\left\{s_{\varepsilon}(a)<z \leqslant s_{0}^{\varepsilon}\right\}}+[a-\varepsilon] g^{\prime \prime \prime}(z)\right\} \\
& =\int_{\varepsilon}^{\min \left\{a, a_{\varepsilon}(z)\right\}}\left[\psi_{z z z}^{\varepsilon}(z, t)-g_{\varepsilon}^{\prime \prime \prime}(z)\right] d t=O(1)\left[\min \left\{a, a_{\varepsilon}(z)\right\}-\varepsilon\right],
\end{aligned}
$$

since $\psi_{z z z}^{\varepsilon}=O(1)\left[1+a^{2}\right]$ in $\mathbf{N}^{\varepsilon}$; see Lemma 4.6 below. This estimate implies the first assertion of the Lemma. It also implies the second assertion since as $z \longrightarrow s_{0}^{\varepsilon}, a_{\varepsilon}(z) \longrightarrow \varepsilon$.

\subsection{Lipschitz continuity of the free boundary}

The key to our analysis is the following estimate:

LEMMA 4.5 Let $M_{0}=\frac{2 \lambda}{(\lambda-1)(R-1-v)}$. There exists a positive constant $M$ depending only on $R$ and $v$ such that $0<-w^{\varepsilon}<\left(M_{0}+M \varepsilon\right)\left(g_{\varepsilon}^{\prime}-\psi_{z}^{\varepsilon}\right)$ in $\mathbf{N}^{\varepsilon}$ and

$$
0<-s_{\varepsilon}^{\prime}(a) \leqslant M_{0}+M \varepsilon, \quad\left(\lambda-1_{\varepsilon}\right) g_{\varepsilon}^{\prime}\left(s_{\varepsilon}(a)\right) \leqslant \ell^{\varepsilon}(a) \leqslant M[1+a] \quad \forall a \in[\varepsilon, T) .
$$

Proof. Set $K=M_{0}+M \varepsilon$ and $\zeta=w^{\varepsilon}+K\left(g_{\varepsilon}^{\prime}-\psi_{z}^{\varepsilon}\right)$. Then $\zeta=0$ on $\Gamma^{\varepsilon}$ and by Lemma $2.5, \zeta \geqslant 0$ on $\left(-\infty, s_{0}^{\varepsilon}\right] \times\{\varepsilon\}$. Using $\mathcal{L}_{1}\left[w^{\varepsilon}\right]=-R w^{\varepsilon}-2 \psi_{z}^{\varepsilon 2}$ and $\mathcal{L}_{1}\left[\psi_{z}^{\varepsilon}\right]=-2 \varphi_{z z}^{\varepsilon} \psi_{z}^{\varepsilon}$ in $\mathbf{N}^{\varepsilon}$ we obtain, in $\mathbf{N}^{\varepsilon}$

$$
\begin{aligned}
\mathcal{L}_{1}\left[w^{\varepsilon}+K\left(g_{\varepsilon}^{\prime}-\psi_{z}^{\varepsilon}\right)\right] & =-R w^{\varepsilon}-2 \psi_{z}^{\varepsilon 2}+K\left[R-1_{\varepsilon}^{2}-v 1_{\varepsilon}+2 \varphi_{z}^{\varepsilon} 1_{\varepsilon}\right] g_{\varepsilon}^{\prime}+2 K \varphi_{z z}^{\varepsilon} \psi_{z}^{\varepsilon} \\
& \geqslant-2 g_{\varepsilon}^{\prime 2}+K\left[R-1_{\varepsilon}^{2}-v 1_{\varepsilon}\right] g_{\varepsilon}^{\prime} \\
& \geqslant\left[K\left(R-1_{\varepsilon}^{2}-v 1_{\varepsilon}\right)-2 g_{\varepsilon}^{\prime}\left(s_{0}^{\varepsilon}\right)\right] g_{\varepsilon}^{\prime}>0 .
\end{aligned}
$$

Hence, by maximum principle, $\zeta>0$, i.e., $0<-w^{\varepsilon}<K\left[g_{\varepsilon}^{\prime}-\psi_{z}^{\varepsilon}\right]$ in $\mathbf{N}^{\varepsilon}$. Consequently, by L'Hôspital rule,

$$
0<-s_{\varepsilon}^{\prime}(a)=\frac{w_{z}^{\varepsilon}\left(s_{\varepsilon}(a), a\right)}{\psi_{z z}^{\varepsilon}\left(s_{\varepsilon}(a), a\right)-g_{\varepsilon}^{\prime \prime}\left(s_{\varepsilon}(a)\right)}=\lim _{z J_{\varepsilon}(a)} \frac{-w(z, a)}{g_{\varepsilon}^{\prime}(z)-\psi_{z}^{\varepsilon}(z, a)} \leqslant M_{0}+M \varepsilon .
$$

Next, using $\psi_{z}^{\varepsilon} \leqslant g_{\varepsilon}^{\prime} e^{\left[\lambda-1_{\varepsilon}\right]\left[z-s_{\varepsilon}(a)\right]}$ we obtain

$$
\begin{aligned}
\ell^{\varepsilon}(a) & =\psi_{z z}^{\varepsilon}\left(s_{\varepsilon}(a), a\right)-g_{\varepsilon}^{\prime \prime}\left(s_{\varepsilon}(a)\right)=\lim _{z \nearrow s_{\varepsilon}(a)} \frac{g_{\varepsilon}^{\prime}(z)-\psi_{z}^{\varepsilon}(z, a)}{s_{\varepsilon}(a)-z} \\
& \geqslant \lim _{z \nearrow_{\gamma_{\varepsilon}}(a)} \frac{g_{\varepsilon}^{\prime}(z)-g_{\varepsilon}^{\prime}(z) e^{\left[\lambda-1_{\varepsilon}\right]\left[z-s_{\varepsilon}(a)\right]}}{s_{\varepsilon}(a)-z}=\left(\lambda-1_{\varepsilon}\right) g_{\varepsilon}^{\prime}\left(s_{\varepsilon}(a)\right) .
\end{aligned}
$$


Also, using $\varphi_{z}^{\varepsilon}=\varphi_{0}^{\varepsilon^{\prime}}+\int_{\varepsilon}^{a} \psi_{z}^{\varepsilon} d t \leqslant O(a) e^{\lambda b}$ we obtain

$$
\ell^{\varepsilon}(a)=R g_{\varepsilon}\left(s_{\varepsilon}(a)\right)+\left[2 \varphi_{z}^{\varepsilon}\left(s_{\varepsilon}(a), a\right)-v\right] g_{\varepsilon}^{\prime}\left(s_{\varepsilon}(a)\right)-g_{\varepsilon}^{\prime \prime}\left(s_{\varepsilon}(a)\right) \leqslant M[1+a] .
$$

This completes the proof.

\section{5 $L^{\infty}$ Estimates}

LEMma 4.6 There exists a positive constant $M$ depending only on $R$ and $v$ such that in $\mathbf{N}^{\varepsilon}$,

$$
\begin{array}{rlrl}
0<\varphi^{\varepsilon}-\varphi_{0}^{\varepsilon} & \leqslant a M e^{\lambda z}, & 0<\varphi_{z}^{\varepsilon}-\varphi_{0}^{\varepsilon} & \leqslant a M e^{\lambda z}, \\
0<\varphi_{z z}^{\varepsilon} & \leqslant a M e^{\lambda z}[1+a], & 0<\varphi_{z z z}^{\varepsilon} & <a M e^{\lambda z}\left[1+a^{2}\right], \\
0<\psi_{0}^{\varepsilon}-\psi^{\varepsilon} & \leqslant a M e^{\Lambda(2 R) z}, & \left|\psi_{0}^{\varepsilon \prime}-\psi_{z}^{\varepsilon}\right| & \leqslant a M e^{\Lambda(2 R) z}[1+a], \\
\left|\psi_{z z}^{\varepsilon}-\psi_{0}^{\varepsilon^{\prime \prime}}\right| & \leqslant a M e^{\Lambda(2 R) z}\left[1+a^{2}\right], & \left|\psi_{z z z}^{\varepsilon}\right| \leqslant M e^{\lambda z}\left[1+a^{2}\right], \\
\left|w^{\varepsilon}-w_{0}^{\varepsilon}\right| \leqslant a M e^{\Lambda(3 R) z}[1+a], & \left|w_{z}^{\varepsilon}\right| \leqslant M e^{\Lambda(2 R) z}[1+a], \\
\left|w_{z z}^{\varepsilon}\right| \leqslant M e^{\Lambda(2 R) z}\left[1+a^{2}\right], & \left|w_{a}^{\varepsilon}\right| \leqslant M e^{\Lambda(3 R) z}[1+a], \\
\left\|s_{\varepsilon}\right\|_{C^{1+1 / 2}([\varepsilon, a])} \leqslant M\left[1+a^{2}\right] .
\end{array}
$$

Proof. 1. First we estimate $w^{\varepsilon}$.

Consider the function $\bar{\zeta}=2 \lambda e^{\Lambda(2 R)(z-b)}-\Lambda(2 R) e^{2 \lambda(z-b)}$. Recalling that $\lambda:=\Lambda(R)<\Lambda(2 R)<$ $2 \lambda$, we see that $\bar{\zeta}_{z}>0$ and $\bar{\zeta}>0$ for $z<b$. Using $\varphi_{z}^{\varepsilon}>0$ we have

$$
\mathcal{L}_{2}[\bar{\zeta}]>-\bar{\zeta}_{z z}-v \bar{\zeta}_{z}+2 R \bar{\zeta}=2 \lambda^{2} \Lambda(2 R) e^{2 \lambda(z-b)}
$$

here we have used $\lambda^{2}+v \lambda=R$ and $\Lambda^{2}(2 R)+v \Lambda(2 R)=2 R$. Since $\mathcal{L}_{2}\left[-w^{\varepsilon}\right]=2 \psi_{z}^{\varepsilon 2}=$ $O(1) e^{2 \lambda z}$, comparing $-w^{\varepsilon}$ with $M \bar{\zeta}$ for suitable large $M$ depending only on $R$ and $v$ we then obtain $0<-w^{\varepsilon}<M \bar{\zeta}=O(1) e^{\Lambda(2 R) z}$ in $\mathbf{N}^{\varepsilon}$.

2. Next, we estimate $\zeta:=w_{z}^{\varepsilon}$.

We have $|\zeta(\cdot, \varepsilon)|=\left|w_{0}^{\varepsilon^{\prime}}\right|=O(1) e^{\Lambda(2 R)(z-b)}$. Also, on $\Gamma^{\varepsilon}$,

$$
|\zeta|=\left|s_{\varepsilon}^{\prime}\right| \ell^{\varepsilon}=O(1)[1+a] .
$$

In $\mathbf{N}^{\varepsilon}$, we have $\mathcal{L}_{21} \zeta:=R a \zeta_{a}+2 R \zeta-\zeta_{z z}+\left(2 \varphi_{z}^{\varepsilon}-v\right) \zeta_{z}+2 \varphi_{z z}^{\varepsilon} \zeta=-4 \psi_{z}^{\varepsilon} \psi_{z z}^{\varepsilon}$. Note that

$$
\left|\psi_{z}^{\varepsilon} \psi_{z z}^{\varepsilon}\right|=\psi_{z}^{\varepsilon}\left|R a w^{\varepsilon}+R \psi^{\varepsilon}+\left(2 \varphi_{z}^{\varepsilon}-v\right) \psi_{z}^{\varepsilon}\right|=O(1)[1+a] e^{2 \lambda z} .
$$

Now consider the function $\bar{\zeta}=[1+a]\left[2 \lambda e^{\Lambda(2 R)(z-b)}-\Lambda(2 R) e^{2 \lambda(z-b)}\right]$. Using $\varphi_{z}^{\varepsilon}>0, \varphi_{z z}^{\varepsilon}>0$, $\bar{\zeta}_{z}>0$ and $\bar{\zeta}_{a}>0$ we obtain, in $\mathbf{N}^{\varepsilon}$,

$$
\mathcal{L}_{21} \bar{\zeta} \geqslant 2 R \bar{\zeta}-\bar{\zeta}_{z z}-v \bar{\zeta}_{z}=2 \lambda^{2} \Lambda(2 R)(1+a) e^{2 \lambda(z-b)} .
$$

Taking a suitable constant $M$ we then see that $M \bar{\zeta}$ is a supersolution and $-M \bar{\zeta}$ is a subsolution; that is, $\left|w_{z}^{\varepsilon}\right| \leqslant M \bar{\zeta}$. Hence, we have $\left|w_{z}^{\varepsilon}\right|=O(1)[1+a] e^{\Lambda(2 R) z}$. 
3. We estimate $v^{\varepsilon}=w_{a}^{\varepsilon}$.

First of all, on $\Gamma^{\varepsilon}, v^{\varepsilon}=\ell^{\varepsilon} s_{\varepsilon}^{\prime 2}=O(1)[1+a]$. Also, initially, $v^{\varepsilon}=v_{0}^{\varepsilon}=O(1) e^{\Lambda(3 R) z}$. In $\mathbf{N}^{\varepsilon}$, we have $\mathcal{L}_{3} v^{\varepsilon}=-6 \psi_{z}^{\varepsilon} w_{z}^{\varepsilon}=O(1)[1+a] e^{[\lambda+\Lambda(2 R)] z}$.

Now consider the function $\bar{\zeta}=[1+a]\left[(\lambda+\Lambda(2 R)) e^{\Lambda(3 R)[z-b]}-\Lambda(3 R) e^{[\lambda+\Lambda(2 R)][z-b]}\right]$. Note that $\Lambda(3 R)<\lambda+\Lambda(2 R)$, so $\bar{\zeta}>0, \bar{\zeta}_{a}>0$, and $\bar{\zeta}_{z}>0$ for $z<b$. In addition, using $\varphi_{z}^{\varepsilon}>0$ we obtain

$$
\mathcal{L}_{3} \bar{\zeta}>3 R \bar{\zeta}-\bar{\zeta}_{z z}-v \bar{\zeta}_{z}=2 \lambda \Lambda(2 R) \Lambda(3 R)[1+a] e^{[\lambda+\Lambda(2 R)][z-b]}
$$

Here we use $\lambda^{2}+v \lambda=R, \Lambda(2 R)^{2}+v \Lambda(2 R)=2 R$, and $\Lambda(3 R)^{2}+v \Lambda(3 R)=3 R$. Thus, we can find a positive constant $M$ such that $M \bar{\zeta}$ is a supersolution and $-M \bar{\zeta}$ is a subsolution. By comparison, $\left|v^{\varepsilon}\right| \leqslant M \bar{\zeta}(z, a)=O(1)[1+a] e^{\Lambda(3 R) z}$.

4. We estimate $\varphi^{\varepsilon}$.

Since $0<\psi^{\varepsilon}=O(1) e^{\lambda z}$ and $0<\psi_{z}^{\varepsilon}=O(1) e^{\lambda z}$, integrating $\varphi_{a}^{\varepsilon}=\psi^{\varepsilon}$ and $\varphi_{z a}^{\varepsilon}=\psi_{z}^{\varepsilon}$ we obtain $0<\varphi^{\varepsilon}-\varphi_{0}^{\varepsilon}=[a-\varepsilon] O(1) e^{\lambda z}$ and $0<\varphi_{z}^{\varepsilon}-\varphi_{0}^{\varepsilon^{\prime}}=[a-\varepsilon] O(1) e^{\lambda z}$. Next from the equation $\varphi_{z z}^{\varepsilon}=R a \psi^{\varepsilon}+\left(\varphi_{z}^{\varepsilon}-v\right) \varphi_{z}^{\varepsilon}$ we obtain $0<\varphi_{z z}^{\varepsilon}=O(1)\left\{a e^{\lambda z}+\left(a e^{\lambda z}\right)^{2}\right\}$. Similarly, using $\varphi_{z z z}^{\varepsilon}=\operatorname{Ra} \psi_{z}^{\varepsilon}+\left(2 \varphi_{z}^{\varepsilon}-v\right) \varphi_{z z}^{\varepsilon}$ we obtain $0<\varphi_{z z z}^{\varepsilon}=O(1)\left\{a e^{\lambda z}+\left(a e^{\lambda z}\right)^{3}\right\}$.

5. We estimate $w^{\varepsilon}$.

Using $w_{z z}^{\varepsilon}=R a v^{\varepsilon}+\left(2 \varphi_{z}^{\varepsilon}-v\right) w_{z}^{\varepsilon}+2 R w^{\varepsilon}+2 \psi_{z}^{\varepsilon 2}$ and $\left|v^{\varepsilon}\right|=O(1)[1+a] e^{\Lambda(3 R) z}$ we obtain $w_{z z}^{\varepsilon}=O(1)\left([1+a] e^{\Lambda(2 R) z}+a^{2} e^{\Lambda(3 R) z}\right)$. Integrating $w_{a}^{\varepsilon}=v^{\varepsilon}$ we obtain $\left|w^{\varepsilon}-w_{0}^{\varepsilon}\right|=O(1)[a-$ $\varepsilon][1+a] e^{\Lambda(3 R) z}$.

6. We estimate $\psi^{\varepsilon}$.

Integrating $\psi_{a}^{\varepsilon}=w^{\varepsilon}, \psi_{z a}^{\varepsilon}=w_{z}^{\varepsilon}$ and $\psi_{z z a}^{\varepsilon}=w_{z z}^{\varepsilon}$ we obtain $0 \leqslant \psi_{0}^{\varepsilon}-\psi^{\varepsilon}=O(1)[a-\varepsilon] e^{\Lambda(2 R) z}$, $\left|\psi_{0}^{\varepsilon^{\prime}}-\psi_{z}^{\varepsilon}\right|=O(1)[a-\varepsilon][1+a] e^{\Lambda(2 R) z}$ and $\left|\psi_{0}^{\varepsilon^{\prime \prime}}-\psi_{z z}^{\varepsilon}\right|=O(1)[a-\varepsilon]\left[1+a^{2}\right] e^{\Lambda(2 R) z}$. Also, using $\psi_{z z z}^{\varepsilon}=R a w_{z}^{\varepsilon}+\left(2 \varphi_{z}^{\varepsilon}-v\right) \psi_{z z}^{\varepsilon}+\left(R+2 \varphi_{z z}^{\varepsilon}\right) \psi_{z}^{\varepsilon}$ we obtain $\psi_{z z z}^{\varepsilon}=O(1)\left[1+a^{2}\right] e^{\lambda z}$.

7. Finally, we estimate $s_{\varepsilon}$.

Set $D_{a}:=\left\{(z, t) \mid t \in[\varepsilon, a], z \leqslant s_{\varepsilon}(t)\right\}$. Since $\left|s_{\varepsilon}^{\prime}\right|=O(1)$, by interpolation, $\left\|w_{z}^{\varepsilon}\right\|_{C^{1,1 / 2}\left(D_{a}\right)}=$ $O(1)\left\{\left\|w_{a}^{\varepsilon}\right\|_{L^{\infty}\left(D_{a}\right)}+\left\|w_{z z}^{\varepsilon}\right\|_{L^{\infty}\left(D_{a}\right)}\right\}=O(1)\left[1+a^{2}\right]$. Consequently, since $s_{\varepsilon}^{\prime}=-w_{z}^{\varepsilon}\left(s_{\varepsilon}, t\right) / \ell^{\varepsilon}$ and $\ell^{\varepsilon}>\lambda-1_{\varepsilon}$, we obtain $\left\|s_{\varepsilon}\right\|_{C^{1+1 / 2}([\varepsilon, a])}=O(1)\left[1+a^{2}\right]$. This completes the proof.

\section{Global existence and proof of Theorem 1}

\subsection{Global existence of (3.1)}

LEMMA 5.1 (Global existence of approximated problem) For every sufficiently small positive $\varepsilon>$ 0 , problem (3.1) with $T=\infty$ admits a solution. The solution is unique in the class of functions in which $w_{z}^{\varepsilon}=O(1)[1+a]$. In addition, $0<-s_{\varepsilon}^{\prime} \leqslant M_{0}+M \varepsilon$ and $0^{\varepsilon}<s_{\varepsilon}<b$ in $[\varepsilon, \infty)$ and for every $\alpha \in(0,1), s_{\varepsilon} \in C^{2+\alpha / 2}([\varepsilon, \infty)) \cap C^{\infty}((\varepsilon, \infty))$ and $w^{\varepsilon} \in C^{3+\alpha,(3+\alpha) / 2}\left(D_{\varepsilon}\right) \cap C^{\infty}\left(D_{\varepsilon} \backslash\left(s_{0}^{\varepsilon}, \varepsilon\right)\right)$, where $D_{\varepsilon}=\left\{(z, a) \mid a \in[\varepsilon, \infty), z \leqslant s_{\varepsilon}(a)\right\}$.

Proof. Let $[\varepsilon, T)$ be the maximum existence interval of (3.1) in which $s_{\varepsilon}^{\prime}<0<\ell^{\varepsilon}$. Suppose $T<\infty$.

Set $\mathbf{N}^{\varepsilon}=\left\{(z, a) \mid a \in(\varepsilon, T), z<s_{\varepsilon}(a)\right\}$ and $\Gamma^{\varepsilon}=\left\{\left(s_{\varepsilon}(a), a\right) \mid a \in[\varepsilon, T)\right\}$. Since $0<-s_{\varepsilon}^{\prime} \leqslant$ $M_{0}+M \varepsilon$, we see that $\Gamma^{\varepsilon}$ is Lipschitz continuous and $s_{\varepsilon}(T):=\lim _{a \nearrow T} s_{\varepsilon}(a)$ exists. Consequently, by parabolic estimates, for every $\alpha \in(0,1)$ there exists a constant $C(\alpha, \varepsilon, T)$ depending on the 
lipschitz norm of $\boldsymbol{\Gamma}^{\varepsilon}$ such that $\left\|w^{\varepsilon}\right\|_{C^{1+\alpha,(1+\alpha) / 2}\left(\mathbf{N}^{\varepsilon}\right)} \leqslant C(\alpha, \varepsilon, T)$. We define

$$
\left(w^{\varepsilon}(\cdot, T), \varphi^{\varepsilon}(\cdot, T), \psi^{\varepsilon}(\cdot, T)\right)=\lim _{a \nearrow T}\left(w^{\varepsilon}(\cdot, a), \varphi^{\varepsilon}(\cdot, s), \psi^{\varepsilon}(\cdot, a)\right) \quad \text { on }\left(-\infty, s_{\varepsilon}(T)\right] .
$$

Since $s_{\varepsilon}$ is Lipschitz continuous, $w^{\varepsilon}=0$ on $\boldsymbol{\Gamma}^{\varepsilon}$, and $w^{\varepsilon}<0$ and $\mathcal{L}_{2} w^{\varepsilon}<0$ in $\mathbf{N}^{\varepsilon}$, by Hopf Lemma, there exists a positive constant $c(\varepsilon, T)>0$ such that $w_{z}\left(s_{\varepsilon}(a), a\right)>c(\varepsilon, T)$ for every $a \in[\varepsilon, T)$. Consequently, $\ell^{\varepsilon}(a)=\frac{w_{z}^{\varepsilon}\left(s_{\varepsilon}(a), a\right)}{-s_{\varepsilon}^{\prime}(a)} \geqslant \frac{c(\varepsilon, T)}{M_{0}+M \varepsilon}$ for every $a \in[\varepsilon, T)$. Thus, from the equation $-s_{\varepsilon}^{\prime}=$ $w_{z}^{\varepsilon}\left(s_{\varepsilon}(a), a\right) / \ell^{\varepsilon}(a)$ we see that $\left\|s_{\varepsilon}^{\prime}\right\|_{C^{\alpha / 2}([\varepsilon, T])} \leqslant C(\alpha, \varepsilon, T)$ and $-s_{\varepsilon}^{\prime} \geqslant \frac{c(\varepsilon, T)}{M[1+T]}$. Once we know the $C^{\alpha / 2}$ regularity of $s_{\varepsilon}^{\prime}$, we find that $\left\|w^{\varepsilon}\right\|_{C^{2+\alpha, 1+\alpha / 2}\left(\overline{\mathbf{N}}^{\varepsilon}\right)} \leqslant C(\alpha, \varepsilon, T)$. A bootstrap argument then show that $s_{\varepsilon} \in C^{\infty}((\varepsilon, T])$ and $w^{\varepsilon} \in C^{\infty}(D)$ where $D=\left\{(z, a) \mid a \in(\varepsilon, T], z \leqslant s_{\varepsilon}(a)\right\}$.

Now taking $T$ as initial time and using $\left(w^{\varepsilon}(\cdot, T), \varphi^{\varepsilon}(\cdot, T), \psi^{\varepsilon}(\cdot, T), s_{\varepsilon}(T)\right)$ as initial data, we can follow the same existence proof presented in the previous section to show that the solution can be extended to $[T+\delta$ ) for some $\delta>0$. But this contradicts the definition that $[\varepsilon, T)$ is the maximum existence interval.

Thus, (3.1) with $T=\infty$ admits a solution. The solution is unique if $w_{z}^{\varepsilon}=O(1)[1+a]$ (cf. the contraction mapping proof in Section 3).

\subsection{The limit process}

We can now send $\varepsilon \searrow 0$ to obtain a solution of (1.6).

1. We extend $\left(\varphi^{\varepsilon}, \psi^{\varepsilon}, w^{\varepsilon}, v^{\varepsilon}, s_{\varepsilon}\right)$ for $z \leqslant b, a \in(0, \varepsilon]$ by

$$
\begin{aligned}
\varphi^{\varepsilon}(z, a) & :=a \psi_{0}+\frac{a^{2}}{2} w_{0}+\frac{a^{3}}{6} v_{0}, \\
\psi^{\varepsilon}(z, a) & :=\frac{1}{a R}\left(\varphi_{z z}^{\varepsilon}+v \varphi_{z}^{\varepsilon}-\varphi_{z}^{\varepsilon 2}\right)=\psi_{0}+a w_{0}+\frac{a^{2}}{2} v_{0}+O\left(a^{3}\right), \\
w^{\varepsilon}(z, a) & :=\frac{1}{a R}\left(\psi_{z z}^{\varepsilon}+\left(v-2 \varphi_{z}^{\varepsilon}\right) \psi_{z}^{\varepsilon}-R \psi_{z}^{\varepsilon}\right)=w_{0}+a v_{0}+O\left(a^{2}\right), \\
v^{\varepsilon}(z, a) & :=\frac{1}{a R}\left(w_{z z}^{\varepsilon}+\left(v-2 \varphi_{z}^{\varepsilon}\right) w_{z}^{\varepsilon}-2 R \psi_{z}^{\varepsilon}+2 \varphi_{z}^{\varepsilon 2}\right)=v_{0}+O(a) . \\
s_{\varepsilon}(a) & :=s_{0}^{\varepsilon}+(a-\varepsilon) s_{1}^{\varepsilon} .
\end{aligned}
$$

We also define $\varphi^{\varepsilon}(\cdot, 0)=0, \psi^{\varepsilon}(\cdot, 0)=\psi_{0}, w^{\varepsilon}(\cdot, 0)=w_{0}, v_{0}^{\varepsilon}(\cdot, 0)=v_{0}$ and $s_{\varepsilon}(0)=s_{0}^{\varepsilon}-s_{1}^{\varepsilon} \varepsilon$.

We remark that this extension provides accurate approximation of the true solution near $a=0$.

2. Since $0^{\varepsilon}<s_{\varepsilon}<b,-\left[M_{0}+M \varepsilon\right] \leqslant s_{\varepsilon}^{\prime}<0$ and $\left\|s_{\varepsilon}\right\|_{C^{1+1 / 2}([0, a])} \leqslant M\left[1+a^{2}\right]$ for every $a>0$, there exists a sequence, $\left\{\varepsilon_{i}\right\}_{i=1}^{\infty}$, of positive real numbers such that $\lim _{i \rightarrow \infty} \varepsilon_{i}=0$ and

$$
\lim _{i \rightarrow \infty} s_{\varepsilon_{i}}(\cdot)=s(\cdot) \quad \text { in } C^{1+\alpha}([0, T]) \quad \forall T>0, \alpha \in(0,1 / 2) .
$$

The limit $s$ satisfies

$$
\begin{gathered}
s(0)=b, \quad s^{\prime}(0)=\frac{\Lambda(2 R)-2 \lambda}{(\lambda-1)^{2} \lambda}, \\
0 \leqslant s(a) \leqslant b, \quad 0 \leqslant-s^{\prime}(a) \leqslant M_{0}, \quad\|s\|_{C^{1+1 / 2}([0, a])} \leqslant M\left[1+a^{2}\right] \quad \forall a>0 .
\end{gathered}
$$


3. Set $W^{\varepsilon}(x, a)=w^{\varepsilon}\left(x+s_{\varepsilon}(a), a\right)$. For each $T>0$ set $D_{T}=(-\infty, 0] \times[0, T]$. Then $\left\|W^{\varepsilon}\right\|_{W_{\infty}^{2,1}\left(D_{T}\right)} \leqslant M\left[1+T^{2}\right]$ where $M$ is a constant depending only on $v$ and $R$. Consequently, (taking a subsequence if necessary), we have

$$
\lim _{i \rightarrow \infty} W^{\varepsilon_{i}}=W \quad \text { in } C^{1+\alpha,(1+\alpha) / 2}((-\infty, 0] \times[0, T]) \quad \forall \alpha \in(0,1), T>0,
$$

where $W$ is a function in $W_{\infty}^{2,1}((-\infty, 0] \times[0, T])$ for any $T>0$.

4. Now define $w(z, a)=W(z-s(a), a)$ for $z \leqslant s(a)$ and $w=0$ for $z>s(a)$. Also define $\psi(z, a)=\psi_{0}(z)+\int_{0}^{a} w(z, t) d t$ and $\varphi(z, a)=\int_{0}^{a} \psi(z, t) d t$ for $(z, a) \in \mathbb{R} \times[0, \infty)$. Then from the differential equation of $w^{\varepsilon}$, we find that

$$
\begin{gathered}
R a w_{a}+2 R w-w_{z z}-v w_{z}+2 \varphi_{z} w_{z}+2 \varphi_{z}^{2}=0 \quad \forall a>0, z<s(a), \\
w(z, a)=0 \quad \forall a \geqslant 0 \quad z \geqslant s(a),
\end{gathered}
$$

$\lim _{a \searrow 0}\left\{\left\|w(\cdot, a)-w_{0}\right\|_{L^{\infty}(\mathbb{R})}+\left\|\psi(\cdot, a)-\psi_{0}\right\|_{L^{\infty}((-\infty, b+1])}+\|\varphi(\cdot, a)\|_{L^{\infty}((-\infty, b+1])}\right\}=0$.

5. $\lim _{i \rightarrow \infty} \ell^{\varepsilon_{i}}(a)=\ell(a):=e^{s(a)}[2 \varphi(s(a), a)+R-1-v]-R$ uniformly in $[0, T]$ for any $T>0$. In addition

$$
\lambda-1 \leqslant \ell(a) \leqslant M[1+a] .
$$

Consequently,

$$
s(a)=b-\int_{0}^{a} \frac{w_{z}(s(t), t)}{\ell(t)} d t \quad \forall a \in[0, \infty) .
$$

It then follows from a bootstrap argument that $s \in C^{1+1 / 2}([0, \infty)) \cap C^{\infty}((0, \infty))$ and $w \in$ $W_{\infty}^{2,1}(D) \cap C^{\infty}(D)$, where $D:=\{(z, a) \mid a>0, z \leqslant s(a)\}$.

6. Once we know that $s \in C^{\infty}((0, \infty))$ and $w \in C^{\infty}(D)$, we can derive the jump relation stated in Theorem 1 from the basic relations $\llbracket \psi \rrbracket=0$ and $\llbracket \psi_{z} \rrbracket=0, \llbracket w \rrbracket=0, \llbracket w_{z} \rrbracket=\ell s^{\prime}$.

We have shown the following:

THEOREM 2 There exists a solution $(\varphi, \psi, w, s)$ of (1.6) satisfying $s \in C^{1+1 / 2}([0, \infty)) \cap$ $C^{\infty}((0, \infty))$ and $w \in W_{\infty}^{2,1}(D) \cap C^{\infty}(D)$ for every $\alpha \in(0,1)$, where $D:=\{(z, a) \mid a>$ $0, z \leqslant s(a)\}$. In addition, the solution satisfies the jump relations stated in Theorem 1 , and in $D$,

$$
\begin{aligned}
& 0 \leqslant \varphi \leqslant a \psi_{0}, \quad 0 \leqslant \varphi_{z} \leqslant a \psi_{0}^{\prime}, \quad 0 \leqslant \varphi_{z z} \leqslant a M e^{\lambda z}[1+a], \\
& \left|\psi-\psi_{0}\right| \leqslant a M e^{\Lambda(2 R) z}, \quad\left|\psi_{z}-\psi_{0}^{\prime}\right| \leqslant a M[1+a] e^{\Lambda(2 R) z},\left|\psi_{z z}-\psi_{0}^{\prime \prime}\right| \\
& \leqslant a M\left[1+a^{2}\right] e^{\Lambda(2 R) z}, \\
& \left|w-w_{0}\right| \leqslant a M[1+a] e^{\Lambda(3 R) z},\left|w_{z}\right| \leqslant M[1+a] e^{\Lambda(2 R) z}, \quad\left|w_{z z}\right| \leqslant M\left[1+a^{2}\right] e^{2 \Lambda(2 R) z}, \\
& 0 \leqslant \varphi_{z z z} \leqslant a M e^{\lambda z}\left[1+a^{2}\right], \quad\left|\psi_{z z z}\right| \leqslant M\left[1+a^{2}\right] e^{\lambda z}, \quad\left|w_{a}\right| \leqslant M[1+a] e^{\Lambda(3 R) z}, \\
& \lambda-1 \leqslant \ell(a) \leqslant M[1+a], \quad\|s\|_{C^{1+1 / 2}([0, a])} \leqslant M\left[1+a^{2}\right] \quad \forall a>0 . \\
& s(0)=b, \quad s^{\prime}(0)=\frac{\Lambda(2 R)-2 \lambda}{(\lambda-1)^{2} \lambda}, \quad 0<s(a)<b, \quad 0<-s^{\prime}(a)=\frac{w_{z}(s(a), a)}{\ell(a)} \leqslant M_{0} .
\end{aligned}
$$




\subsection{Continuity of $w_{z z}$ and $w_{a}$ at $a=0$}

LEMMA $5.2 w \in C^{2,1}(\bar{D})$ and $w_{a}(\cdot, 0)=v_{0}(\cdot)$ on $(-\infty, b]$.

Proof. (1) Fix $a \in(0,1)$. Set $\zeta(z)=w(z, a)-w_{0}(z)$. Then $\zeta(-\infty)=0$ and $\zeta(s(a))=$ $-w_{0}(s(a))$. Also, using $w_{0}^{\prime \prime}+v w_{0}^{\prime}-2 R w_{0}=2 \psi_{0}^{\prime 2}$ we obtain, in $(-\infty, s(a))$,

$$
\zeta^{\prime \prime}+v \zeta^{\prime}-2 R \zeta=R a w_{a}+2 \varphi_{z} w_{z}+2\left(\psi_{z}-\psi_{0}^{\prime}\right)\left(\psi_{z}+\psi_{0}^{\prime}\right)=O(a) e^{\lambda z}
$$

here we use the fact that $\varphi_{z}=O(a)$ and $\psi_{z}(z, a)-\psi_{0}^{\prime}(z)=\psi_{z}(z, a)-\psi_{z}(z, 0)=$ $O(1) a\left\|w_{z}\right\|_{L^{\infty}}=O(1) a$. It then follows from elliptic estimate that

$$
\left\|w(\cdot, a)-w_{0}(\cdot)\right\|_{C^{2}((-\infty, s(a)])}=\|\zeta\|_{C^{2}((-\infty, s(a)])}=O\left(a+\left|w_{0}(s(a))\right|\right)=O(1) a .
$$

(2) Set $D_{\varepsilon}=\left\{(z, a) \mid a \in[\varepsilon, 1], z \leqslant s_{\varepsilon}(a)\right\}$. Using $\left\|w_{z}^{\varepsilon}\right\|_{C^{1,1 / 2}\left(D_{\varepsilon}\right)}=O(1)$ and $v_{0}^{\prime \prime}+v v_{0}^{\prime}-$ $3 R v_{0}=6 \psi_{0}^{\prime} w_{0}^{\prime}$ one finds that

$$
\mathcal{L}_{3}\left(v^{\varepsilon}-v_{0}\right)=6\left[\psi_{0}^{\prime} w_{0}^{\prime}-\psi_{z}^{\varepsilon} w_{z}^{\varepsilon}\right]-2 \varphi_{z}^{\varepsilon} v_{0}^{\prime}=O(\varepsilon+\sqrt{a}) e^{\lambda z}=O(\sqrt{a}) e^{\lambda z} .
$$

Also, using $v_{0}^{\varepsilon}-v_{0}=O(\varepsilon) e^{\lambda z}=O(a) e^{\lambda z}, v^{\varepsilon}=\ell^{\varepsilon} s_{\varepsilon}^{\prime 2}$ on $\Gamma^{\varepsilon}$ and $\left\|s_{\varepsilon}^{\prime}\right\|_{C^{1 / 2}([\varepsilon, 1])}=O(1)$ we find that $\left|v^{\varepsilon}-v_{0}\right|=O(\sqrt{a}+\varepsilon)=O(1) \sqrt{a} e^{\lambda z}$ on the parabolic boundary of $D_{\varepsilon}$. Comparing $\pm\left(v^{\varepsilon}-v_{0}\right)$ with $M \sqrt{a} e^{\lambda z}$ we then find that $\left|v^{\varepsilon}-v_{0}\right|=O\left(\sqrt{a} e^{\lambda z}\right)$. Sending $\varepsilon \searrow 0$ we conclude that

$$
\left\|w_{a}(\cdot, a)-v_{0}(\cdot)\right\|_{C((-\infty, s(a)])}=O(\sqrt{a}) .
$$

Since $w \in C^{\infty}(D) \cap W_{\infty}^{2,1}(D)$, the above estimates imply that $w \in C^{2,1}(\bar{D})$ and $w_{a}(\cdot, 0)=v_{0}(\cdot)$ on $(-\infty, b]$.

\subsection{The variational property}

First of all, we have $\psi_{z}<g^{\prime}$ in $\mathbf{N}$. By integration we find that

$$
\psi(z, a)-g(z, a)=\int_{z}^{s(a)}\left[g^{\prime}(y)-\psi_{y}(y, a)\right] d y>0 \quad \forall(z, a) \in \mathbf{N} .
$$

Also, since $\psi=g$ for $z \geqslant s(a)$, we have, when $z>s(a)$,

$$
R a \psi_{a}-\psi_{z z}+\left(2 \varphi_{z}-v\right) \psi_{z}+R \psi=\left[2 \varphi_{z}(z, a)+R-1-v\right] e^{z}-R=l(z, a) .
$$

Note that $l$ is a continuous function. In addition, $l(s(a), a)=\ell(a)>\lambda-1>0$. Also, since $\varphi_{z z} \geqslant 0$ (c.f. Lemma 4.3) we see that $l_{z}(z, a)>0$ for $z \in \mathbb{R}$. Hence, $l(z, a) \geqslant \lambda-1$ for all $a \geqslant 0, z \geqslant s(a)$. Thus,

$$
\min \left\{\mathcal{L}_{1}[\psi], \psi-g\right\}=0 \text { in } \mathbb{R} \times(0, \infty), \quad \psi(\cdot, 0)=\psi_{0} .
$$

Integrating $\mathcal{L}_{1}[\psi]=l(z, a) \mathbf{1}_{\{z>s(a)\}}$ we find that

$$
Q[\varphi]=\eta:=\int_{0}^{a} l(z, t) \mathbf{1}_{\{s(t)<z\}} d t=\int_{\min \left\{a, a_{*}(z)\right\}}^{a} l(z, t) d t,
$$


where $a=a_{*}(z)$ is the inverse function of $z=s(a)$ for $z \in(0, b), a_{*}(z)=0$ for $z>b$ and $a_{*}(z)=\infty$ for $z \leqslant 0$. As $a_{*}$ and $l$ are continuous, we see that $\eta$ is continuous. This implies that $\varphi \in C^{2}(\mathbb{R} \times[0, \infty))$ and

$$
\min \{\mathbb{Q}[\varphi], \mathbb{B} \varphi\}=0 \text { in } C(\mathbb{R} \times[0, \infty)), \quad \varphi(\cdot, 0)=0 .
$$

Hence, $\varphi$ is a solution of (1.1). In addition, by the uniqueness result of [10], such $\varphi$ is unique. This means that the solution of (1.6) is unique.

This completes the proof of Theorem 1.

REMARK 5.1 Similar to the Stefan Problem, the equation for $w$ can be written as

$$
\begin{aligned}
\frac{\partial}{\partial a}\left(R a w+L(z, a) \mathbf{1}_{\{w<0\}}\right)-w_{z z}-v w_{z}+R w=0 & \text { in } \mathbb{R} \times(0, \infty), \\
w(\cdot, 0)=w_{0} & \text { on } \mathbb{R} \times\{0\} .
\end{aligned}
$$

Here $e(w):=\operatorname{Raw}+L(z, a) \mathbf{1}_{\{w<0\}}$ can be regarded as the enthalpy (i.e., internal energy) and $L(z, a)=2 \varphi_{z} \psi_{z}+(R-1-v) e^{z}-R$ is the latent heat. This is a solidification process of undercooled liquid, the region $[s(a), \infty)$ is solid and $(-\infty, s(a))$ is undercooled liquid.

Acknowledgements. Qin and Lai acknowledge support from CSC. Chen thanks the support from NSF grant DMS-1008905.

\section{REFERENCES}

1. Carpenter, J.N., The exercise and valuation of executive stock options, J. Financial Economics $\mathbf{4 8}$ (1998), 127-158.

2. Carpenter, J.N., Stanton, R. \& Wallace, N., Optimal exercise of executive stock options and implications for firm cost, J. Financial Economics 98 (2010), 315-337.

3. Chen, X. \& DAI, M., Characterization of optimal strategy for multi-asset investment and consumption with transaction costs, SIAM J. Financ. Math. 4 (2013), 857-883, electronic only. Zbl1283.91165 MR3131476

4. Chen, X., Hu, B. \& Liang, J., Finite difference scheme convergence rate for approximated free boundaries of American options, preprint.

5. Friedman, A., Variational Principles and Free Boundary Problems, John Wiley \& Sons, New York, 1982. Zb10564. 49002 MR0679313

6. Grasselli, M. \& Henderson, V., Risk aversion and block exercise of executive stock options, Journal of Economics Dynamics \& Control 33 (2009), 109-127. Zbl1170.91413 MR2477679

7. HAll, B. J. \& Murphy, K. J., Stock option for undiversified executives, J. Accounting and Economics 33 (2002) 3-42.

8. Ingersoll, J., The subjective and objective evaluation of incentive stock options, J. Business 79 (2006) 453-487.

9. Jain, A. \& Subramanian, A., The intertemporal exercise and valuation of employee options, The Accounting Review 79 (2004), 705-743.

10. LAI, X., Chen, X., QIn, C. \& YU, W., Mathematical analysis of a variational inequality modeling perpetual executive stock options, Euro. J. of Applied Mathematics 26 (2015), 193-213.

11. LAI, X., CHEN, X., QIN, C. \& YU, W., A variational inequality for optimal exercise perpetual executive stock options, preprint. 
12. Lambert, R., Larcker, D. \& Verrecchia, R., Portfolio considerations in valuing executive compensation, Journal of Accounting Research 29 (1991) 129-149.

13. Leung, T. \& SiRCAR, R., Accounting for risk aversion, vesting, job termination risk and multiple exercises in valuation of employee stock options, Mathematical Finance 19 (2009), 99-128. Zbl1155. 91388 MR2488404

14. QIn, C., Chen, X., LaI, X. \& YU, W., The optimal exercise strategy for perpetual ESOs under continuous-exercise model, preprint., preprint.

15. Rogers, L. C. G. \& Scheinkman, J., Optimal exercise of executive stock options, Finance Stoch 11 (2007), 357-372. Zbl1164.62084 MR2322917

16. Song, L. \& YU, W., A parabolic variational inequality related to the perpetual American executive stock options, Nonlinear Analysis 74 (2011), 6583-6600. Zbl1254.35222 MR2834063

17. Song, L. \& YU, W., A free boundary problem coming from the perpetual American call options with utility, Euro. J. Appl. Math. 24 (2013), 231-271. Zb106184178 MR3031779 